\title{
The neighbourhood physical environment and active travel in older adults: a systematic review and meta-analysis
}

\author{
Ester Cerin ${ }^{1,2,3^{*}}$ (D), Andrea Nathan ${ }^{1}$, Jelle van Cauwenberg ${ }^{4}$, David W. Barnett', and Anthony Barnett ${ }^{1}$ \\ on behalf of the Council on Environment and Physical Activity (CEPA) - Older Adults working group
}

\begin{abstract}
Background: Perceived and objectively-assessed aspects of the neighbourhood physical environment have been postulated to be key contributors to regular engagement in active travel (AT) in older adults. We systematically reviewed the literature on neighbourhood physical environmental correlates of AT in older adults and applied a novel meta-analytic approach to statistically quantify the strength of evidence for environment-AT associations.

Methods: Forty two quantitative studies that estimated associations of aspects of the neighbourhood built environment with AT in older adults (aged $\geq 65$ years) and met selection criteria were reviewed and meta-analysed. Findings were analysed according to five AT outcomes (total walking for transport, within-neighbourhood walking for transport, combined walking and cycling for transport, cycling for transport, and all AT outcomes combined) and seven categories of the neighbourhood physical environment (residential density/urbanisation, walkability, street connectivity, access to/availability of services/destinations, pedestrian and cycling infrastructure, aesthetics and cleanliness/order, and safety and traffic).
\end{abstract}

Results: Most studies examined correlates of total walking for transport. A sufficient amount of evidence of positive associations with total walking for transport was found for residential density/urbanisation, walkability, street connectivity, overall access to destinations/services, land use mix, pedestrian-friendly features and access to several types of destinations. Littering/vandalism/decay was negatively related to total walking for transport. Limited evidence was available on correlates of cycling and combined walking and cycling for transport, while sufficient evidence emerged for a positive association of within-neighbourhood walking with pedestrian-friendly features and availability of benches/sitting facilities. Correlates of all AT combined mirrored those of walking for transport. Positive associations were also observed with food outlets, business/institutional/industrial destinations, availability of street lights, easy access to building entrance and human and motorised traffic volume. Several but inconsistent individual- and environmental-level moderators of associations were identified.

Conclusions: Results support strong links between the neighbourhood physical environment and older adults' AT. Future research should focus on the identification of types and mixes of destinations that support AT in older adults and how these interact with individual characteristics and other environmental factors. Future research should also aim to clarify dose-response relationships through multi-country investigations and data-pooling from diverse geographical regions.

Keywords: Older adults, Active travel, Cycling, Walking, Neighbourhood, Built environment, Meta-analysis, Systematic review, Moderators

\footnotetext{
* Correspondence: ecerin@hku.hk; Ester.Cerin@acu.edu.au

${ }^{1}$ Institute for Health and Ageing, Australian Catholic University, Level 6, 215 Spring Street, Melbourne, VIC 3000, Australia

${ }^{2}$ School of Public Health, The University of Hong Kong, Hong Kong, China

Full list of author information is available at the end of the article
} 


\section{Background}

Being physically active in old age is associated with numerous positive health outcomes, such as lower incidence of cognitive impairment, depression, dementia [1], coronary heart disease, some types of cancers, diabetes, stroke and hypertension [2]. The risk of all-cause mortality has been shown to be reduced by walking and cycling even after adjustment for other physical activity (PA), with the greatest impact from the first $120 \mathrm{~min}$ and 100 min per week for walking and cycling, respectively [3]. Active travel (AT), here defined as walking or cycling to a destination, can contribute substantiality to the accumulation of health-enhancing levels of PA in older adults. Older adults from Hong Kong, Chicago (USA) and Ghent (Belgium) reported AT accounted for $55 \%$ (169 $\mathrm{min}$ ) of walking within the neighbourhood, $56 \%(159 \mathrm{~min})$ of total walking and $42 \%$ (123 $\mathrm{min})$ of total PA, respectively [4-6]. Changes in active commuting have been associated with corresponding changes in total PA without compensatory changes in leisure-time PA, suggesting a net benefit from engaging in AT [7, 8]. Furthermore, older people appear to experience greater overall health benefits from transport mode shifts to AT than younger people [9].

Benefits in addition to contributions to total PA are also incurred from AT. These include a quieter environment due to decreased motor vehicle noise as well as reductions in greenhouse gas emissions, air pollution, traffic congestion and transport costs [10]. Modelling has shown replacement of urban trips in private motor vehicles with AT can result in important health and economic benefits and reductions in pollutants [11-13]. Furthermore, while AT may increase exposure to air pollutants, PA benefits of AT with respect to all-cause mortality outweighed the harm caused by all but extreme levels of air pollution [14].

As opposed to planned PA (exercise), AT is typically incidental and not specifically accrued to enhance physical fitness. That is, by definition the goal of AT is to reach a destination, not to accumulate PA. AT may, therefore, not be as influenced by individual-level barriers related to participation of older adults in organised PA, such as affordability, lack of self-confidence, social awkwardness, cultural sensitivity and disinterest in PA [15].

The prevalence of PA in older adults in many countries is low [16-18]. Therefore, from a public health perspective, there is a pressing need to identify factors that can positively affect AT participation in this demographic at a population level. Perceived and objectively-assessed aspects of the neighbourhood physical environment have been postulated to be key contributors to regular engagement in AT [19]. They are defined as the objective and perceived characteristics of the physical context in which people spend their time (e.g., home, neighbourhood), including aspects of urban design (e.g., presence of sidewalks), traffic volume and speed, distance to and design of venues for PA (e.g., parks) and other destinations, crime and safety [20].

As the distance older adults travel from their home during their daily life typically decreases with age [21], the neighbourhood environment (rather than the environment around other locations) becomes increasingly important to this cohort. Also, environmental characteristics within $500 \mathrm{~m}$ from home appear to be more predictive of PA for older adults than those within 1-1.6 km typically used for younger age groups [22], further supporting a reduction in life space during old age. Neighbourhood characteristics facilitating engagement in AT are likely to have a large-scale, population-level effect on PA in this population. Their identification is therefore important to inform urban planning interventions.

A 2011 systematic review of associations between the physical environment and PA in older adults identified only six studies on walking for transportation and no studies on cycling for transportation [19]. Also, all these findings were from highly economically developed and low-to-medium density countries. As the number of studies has increased substantially in the last 5 years, we aimed to systematically review the literature on neighbourhood physical environmental correlates of AT in older adults. In doing so, we introduced several methodological improvements in line with the socio-ecological model of active living proposed by Sallis et al. [23], which emphasises the importance of an interactional, contextspecific and domain-specific approach. For example, because environmental correlates of walking for transport appear to differ from those of cycling for transport $[6,24,25]$, we examined the evidence by type of AT as well as for all types of AT combined. We also evaluated article quality and, where appropriate, reported and examined findings stratified by type of environmental measures (self-reports vs. objective) and noted moderators of environment-AT associations [26].

As noted in previous systematic reviews of environment correlates of PA $[19,20]$, studies in this research field typically used a variety of measures of environmental exposures and AT outcomes that precluded the conduct of a traditional meta-analysis with exact quantification of effect sizes. Consequently, previous reviews adopted an exclusively descriptive approach to the synthesis of findings relying on 'subjective' conclusions based on the comparison of the number of positive, zero and negative associations. This typically resulted in authors stating that there was no evidence of a relationship in cases when the frequencies of positive or negative associations were equal to or slightly lower than the frequency of nil associations. In contrast, in addition to providing a descriptive analysis of findings, this review incorporated article/ 
study quality and sample size information into a meta-analytic approach statistically quantifying the strength of evidence for environment-AT associations. Specifically, we adapted established meta-analytic procedures to estimate the probability of observing a certain distribution of positive, negative and nil associations under a null hypothesis of no associations, therefore, providing a more robust, statistically evidence-based synthesis of findings.

\section{Methods}

Details of the protocol for this systematic review and meta-analysis were registered in PROSPERO (Registration no. CRD42016046818 [27]).

\section{Search strategy, study selection and inclusion criteria}

Although this particular systematic review focuses on environmental correlates of older adults' AT (cycling and walking for transport) only, the initial literature search aimed to provide an expanded update of Van Cauwenberg et al.'s systematic review on environmental correlates of all types of PA in older adults [19]. Van Cauwenberg et al.'s original search strategy [19] was broadened to include all types of study design, written material (grey literature) other than peer-reviewed journal articles (e.g., technical reports, proceedings, case reports and government publications) and additional key terms describing environmental exposures (i.e., physical characteristics, physical attributes, urban form, urban design, built form, greenness, park, parks and open space). A complete list of key search terms is given in Fig. 1, as they were used in PubMed. These search terms were customised for each database.

Systematic searches were performed in the following six electronic databases covering the period from January 2000 (as per Van Cauwenberg et al.'s earlier systematic review [19]) to 6th September 2016: CINAHL, PubMed, Scopus, SportDiscus, TRID and Web of Science. Additionally, a purposive sample of relevant websites was searched for grey literature (Active Living Research, SUSTRANS, National Institutes of Health, Clinical Excellence and Heart Foundation and Open Grey) and extant systematic reviews, meta-analyses and authors' personal archives were manually screened. The searches were limited to articles in English. Three reviewers independently screened articles' titles and abstracts for inclusion and discussed undecided cases and disagreements. Each article obtained

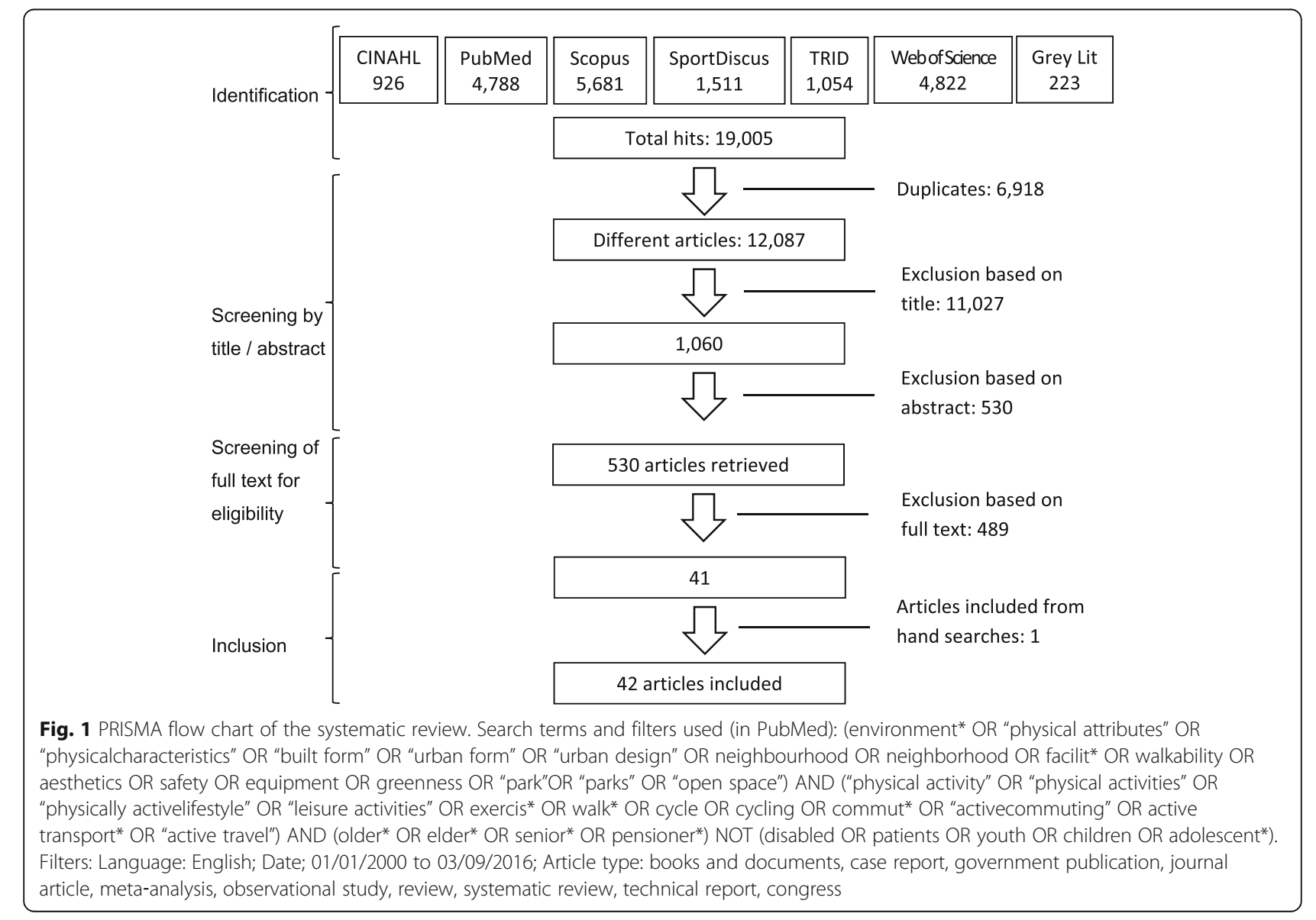


in full text was then independently reassessed for eligibility by two reviewers and categorised according to PA outcomes into AT, leisure-time PA, total PA and/or total (not domain-specific) walking. Undecided cases and discrepancies were assessed by one or two additional reviewers and discussed with the whole team. After selecting eligible articles on AT, we re-examined the six electronic databases and Google Scholar for additional relevant output (meeting the selection criteria) by the first authors of selected articles, whereby authors' names and their affiliations were used as search terms. We also screened the reference lists of selected articles.

Articles were included if they (a) quantitatively investigated the association between any objective or perceived attribute of the neighbourhood physical environment and a measure of AT (engagement in, frequency and/or amount of walking and/or cycling for transport); and (b) had a sample with a mean age of $\geq 65$ years or conducted separate analyses in subsamples with a mean age of $\geq 65$ years. We used 65 years as a cut off value because this was in line with Van Cauwenberg et al.'s review [19] and corresponded to the conventional definition of 'older' adults based on the qualifying state pension age in many developed countries. Articles were excluded if they (a) exclusively focused on a specific clinical population (i.e., only overweight, disabled or institutionalised participants); and/or (b) solely examined associations of AT with an ill-defined composite measure of the neighbourhood environment (e.g., studies using a study-specific, not wellestablished environmental index combining various poorly-correlated aspects of the built environment, such as safety and land use). Figure 1 depicts a flow chart of the systematic literature search following PRISMA guidelines [28] (see Additional file 1).

\section{Data extraction and validity assessment Information extracted}

For each included article, two reviewers extracted data, assessed study quality, verified each other's work and resolved any discrepancies by discussion with a third reviewer and, when necessary, by contacting the authors. Data extraction was conducted using a piloted form (table) with written instructions detailing the type of information to be extracted and how to record, categorise or code it. The following information was extracted from each manuscript: (a) study name; (b) first author and year of publication; (c) sample characteristics (sample size, urban or rural location, age group, sex distribution, response rate and geographical location); (d) study design (type of study, sampling strategy for selection of study areas and participants, and neighbourhood definition); (e) list of covariates included in the analyses; (f) outcome measures (instrument, operationalisation and whether it was a validated or commonly-used measure); (g) environmental exposure measures (objective or perceived measures, attribute measured); (h) list of moderators (if any) and breakdown of sample size by categorical moderator; (i) analytical approach (type of analysis, adjustment for neighbourhood-level clustering, appropriateness of analytical approach); (j) results (associations and moderating effects); and (k) additional comments assisting the quality assessment or interpretation of the article (Additional file 2).

For analytical purposes, AT variables were categorised into: (a) total walking for transport; (b) within-neighbourhood walking for transport; (c) cycling for transport; and (d) total AT (representing measures combining walking and cycling for transport). Environmental variables were classified into categories primarily corresponding to those of the Neighborhood Environment Walkability Scale (NEWS), the most popular measure of perceived neighbourhood environmental attributes worldwide [29-31], which were complemented by several additional attributes appearing in the selected articles. Environmental variables encompassed: (a) walkability, denoting a composite index including information on access to services/land use mix, residential density and/or street connectivity (b) residential density/urbanisation; (c) street connectivity; (d) access to/ availability of services with the subcategories (d.1) overall access to destinations, (d.2) land use mix - destination diversity, (d.3) shops/commercial destinations, (d.4) food outlets, (d.5) business/government/institutional/industrial destinations, (d.6) health and age-care destinations, (d.7) religious destinations, (d.8) public transport, (d.9) park/ open space/recreational destinations, (d.10) entertainment and (d.11) other destinations; (e) streetscape and pedestrian and cycling infrastructure with the subcategories (e.1) pedestrian-friendly features, (e.2) barriers to walking/cycling, (e.3) benches/sitting facilities, (e.4) streetlights, (e.5) easy access to building entrance and (e.6) public toilets; (f) aesthetics and cleanliness/order with the subcategories (f.1) greenery and aesthetically pleasing scenery, (f.2) littering/ vandalism/decay and (f.3) pollution (air/noise); (g) safety and traffic with the subcategories (g.1) traffic/pedestrian safety, (g.2) human and motorised traffic volume, and (g.3) crime/personal safety.

\section{Coding and quantification of findings}

Relationships between physical environmental variables and the AT outcomes were categorised as significantly positive $(\mathrm{P})$, significantly negative $(\mathrm{N})$ or not statistically significant $(\varnothing)$. Single articles were allowed to contribute with more than one finding (association) to a specific combination of environmental attribute and AT outcome if they had more than one distinct environmental variable and/or AT outcomes falling in the same categories. For example, Barnett and colleagues [4] reported two 
associations between land use mix - destination diversity and within-neighbourhood walking for transport - one for walking frequency, the other for volume (i.e., weekly minutes) of walking. These counted as two distinct findings (Additional file 2). Cain et al. [32] reported associations between AT and three aspects of the environment classified under the category 'greenery and aesthetically pleasing scenery' (trees; building aesthetic/design and positive aesthetic and social characteristics). These counted as three distinct findings (Additional file 2).

To avoid duplication of data, study findings reported by more than one article were included only if they represented original information (Additional file 2). If findings from the same study appeared in more than one article, preference was first given to those adjusted for self-selection (if available) and then to those unadjusted for other environmental variables but adjusted for sociodemographic confounders. Studies reporting multiple associations for the same combination of environmental attribute and AT outcome by different area sizes (e.g., buffers of different sizes or areas representing retirement villages vs. neighbourhoods around villages) had fractional weights assigned to each finding so that the sum of the weights across all examined associations was 1 . For example, Etman et al. [33] reported nil associations between walking and aesthetics for $400 \mathrm{~m}$ and $1.6 \mathrm{~km}$ buffers around the home, while they observed positive associations for $800 \mathrm{~m}$ and $1.2 \mathrm{~km}$ buffers. In this case, each of the four findings was assigned a weight of 0.25 , resulting in a summary score of 0.5 nil and 0.5 positive associations.

Studies that found significant moderators of environment-AT associations had associations reported as main effects only if the associations across all examined values of the moderator were consistent in direction and statistical significance (see King et al., [34] in Additional file 2 ). If this was not the case, associations at each examined value of the moderator were assigned fractional weights corresponding to the (approximate) proportion of the total sample represented by the subgroup of participants. For example, Inoue et al. [35] observed negative associations between traffic safety and walking for transport in men but not in women (i.e., sex was a moderator) (Additional file 2). As the sample consisted of $51 \%$ men, the negative association was assigned a weight of 0.51 , while the nil association in women was assigned a fractional weight of 0.49. For continuous moderators, associations computed at the average value of the moderator were assigned a weight of 0.60 , while those at 1 standard deviation (SD) below and above the mean were each assigned a weight of 0.20 (the total sum of the weights is 1 ). The logic behind this is that, under the Normal distribution, the proportions of values 1SD above and below the mean are $\sim 20 \%$ (factoring some uncertainty around the value of the moderator at $+1 S D$ and -1SD). If an association was moderated by multiple factors, weights were assigned following the logic described above but in such a fashion that the sum of the weights across all examined values of all the significant moderators was 1 . The weighting procedure described above was used to estimate the main effects of environmental attributes on AT, while moderators (e.g., sex and age) of associations between environmental factors and AT outcomes were examined and summarised in separate analyses.

\section{Quality and sample size assessment}

Article quality was assessed using nine criteria mirroring those used in other systematic reviews of environmental correlates of health-related and transportation behaviours $[36,37]$, and taking into account other methodological considerations relevant to this research field. These quality criteria included: (1) stratification of neighbourhoods or participants by key environmental attributes to maximise variability in exposures and outcomes [30, 38]; (2) sample shown to be representative of the population or response rate $\geq 60 \%$ [37]; (3) AT outcome measure shown to be valid or representing commonly-used measure [37]; (4) adjustment for socio-demographic covariates (at least age, sex, education or similar) [37]; (5) adjustment for selfselection into neighbourhoods [36]; (6) analytical approach accounted for area-level clustering (if appropriate) [39]; (7) analytical approach correctly accounted for distributional assumption of AT outcome; (8) analyses conducted and presented correctly (i.e., formal testing of moderators, if applicable; presentation of point estimates and 95\% confidence intervals, standard errors and/or $p$-values); and (9) did not inappropriately categorise continuous environmental exposure [40]. Items 1-5 and 9 were each assigned a score of 1 , while the three items (6-8) pertaining to statistical analyses were each assigned a score of $1 / 3$ (i.e., 0.33). The latter was done to avoid overstating the importance of the statistical aspect of the article [by assigning a maximum of 3 rather than 1 point $(0.33 * 3)$ to statistical issues] compared to other methodological issues (e.g., measurement; sample representativeness; internal validity; study design). We did not include an item assessing quality of study design in terms of strength of evidence of causality (cross-sectional, longitudinal and quasi-experimental design) because all studies included in the review were cross-sectional (Table 1). Scores on the above items were summed to yield an overall article quality score ranging from 0 to 7 . Articles with a score ranging from 0 to 3.5 were deemed to be of low quality, those scoring from 3.6 to 5.9 were considered of moderate quality, and those scoring 6 to 7 were deemed high quality.

Articles were also assigned a score for sample size. The total article quality score and a sample-size score 
Table 1 Characteristics of the selected articles/studies $(N=42)$

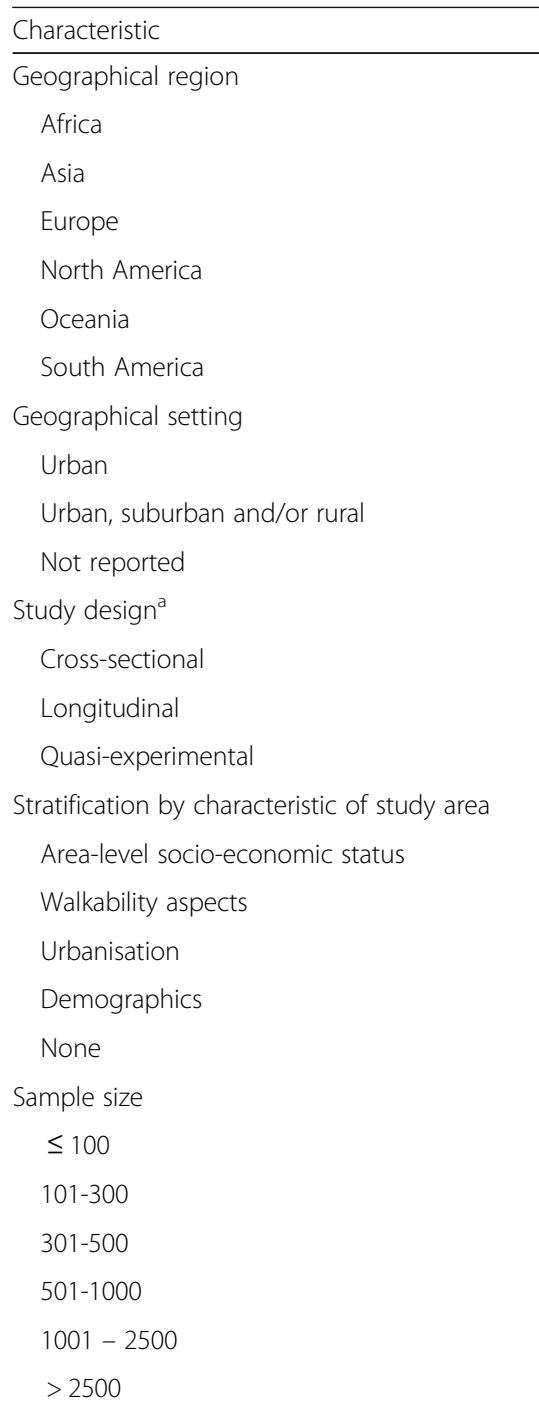

Neighbourhood definition ${ }^{a}$

Objective

Administrative/census area

Buffer (crow-fly or road-network)

400-500 m

$\geq 1000 \mathrm{~m}$

variable/not fixed Number of articles \%

1

6

9

18

5

3

Perceived

10-20 min walk from home

Other participant delimitation

Unknown

Studies with multiple publications

\section{SNQLS}

Active Living Study

BEPAS Seniors

EpiFloripa Elderly
Table 1 Characteristics of the selected articles/studies $(N=42)$ (Continued)

HK Elderly 1
Montreal's Household Travel Survey
Single publication from studies with name
Single publication from studies with no
name
Environmental attributes measured ${ }^{\text {a }}$
Residential density/urbanisation
Objectively assessed
Perceived
Walkability
Objectively assessed
Perceived
Street connectivity
Objectively assessed
Perceived
Access to/availability of services and
destinations
Objectively assessed
Perceived
Pedestrian \& cycling infrastructure
Objectively assessed
Perceived
Aesthetics and cleanliness/order
Perceived
Perceived assessed
Objectively assessed
Objaffic

7.1

Active travel measures ${ }^{a}$ (all self-reported)

Total walking for transport ${ }^{c}$

Continuous - frequency

Continuous - amount

Categorical - any, yes/no

Categorical $-60+$ min/week, yes/no

Categorical - 150+ min/week, yes/no

Categorical - daily, yes/no

Categorical - 3 categories/levels

Within-neighbourhood walking for transport ${ }^{\mathrm{c}}$

$\begin{array}{ll}\text { Continuous - frequency } & 2 \\ \text { Continuous - amount } & 4\end{array}$


Table 1 Characteristics of the selected articles/studies $(N=42)$ (Continued)

\begin{tabular}{lll}
\hline Active travel (walking + cycling) & 5 & 11.9 \\
Continuous - frequency & 1 & 2.4 \\
Continuous - amount & 3 & 7.1 \\
Categorical - 3 categories/levels & 1 & 2.4 \\
Moderators of environment-active travel associations ${ }^{\mathrm{a}}$ & \\
Individual & & \\
Socio-demographics & 6 & 14.3 \\
Psychosocial factors & 1 & 2.4 \\
Vehicle ownership or driving status & 1 & 2.4 \\
Health status/functionality & 3 & 7.1 \\
Environmental & & \\
Area-level income & 4 & 9.5 \\
Residential density/urbanisation & 2 & 4.8 \\
Pedestrian infrastructure & 1 & 2.4 \\
Safety and traffic & 2 & 4.8 \\
None & 25 & 59.5 \\
\hline
\end{tabular}

${ }^{a}$ Multiple options allowed in single articles

${ }^{b}$ One article had both objective and perceived measures of access to/availability of services and destinations. Hence, the total number of articles is 1 unit smaller than the sum of articles with objectively assessed and perceived measures 'Some articles had more than one measure of walking. Hence, the total number of articles is smaller than the sum of articles with specific measures of walking

were used to compute an 'article weight' for the metaanalytical procedure described below. The following weights were assigned: 0.25 for a sample of $\leq 100$ participants; 0.50 for $101-300$ participants; 1.00 for $301-500$ participants; 1.25 for $501-1000$ participants; 1.50 for 1001-2500 participants; and 1.75 for $>2500$ participants. A weight of 1.00 was assigned to studies with $301-500$ participants as these are 'typical' sample sizes in this field and, also, adequate to examine small-to-moderate associations (based on sample size calculations) [30, 41]. Non-linearly incremental weights were assigned to sample sizes because the gain in statistical power is the greatest for smaller samples and decreases as the sample size increases [42]. The sample size categories were capped at 2,501+ participants because such a sample size allows the detection of a very small effect equivalent to $1 \%$ of outcome variance explained even in presence of a substantial residual clustering effect (intra-class correlation of 0.10 ).

\section{Data synthesis}

Summary of findings were given in the form of number of positive, negative and statistically non-significant associations by each combination of environmental attribute - AT outcome (e.g., shops/commercial destinations - cycling for transport) included in this review. In addition, associations were also tallied for all AT outcomes, i.e., irrespective of the type of AT, to provide a general conclusion regarding the effects of neighbourhood environmental attributes on AT. The tallying process took into account the fractional weights assigned to findings from studies examining associations by different area sizes and values of the significant moderators.

To assist the interpretation of findings and conclusions, we used a meta-analytic approach to derive conservative estimates of p-values for each combination of environmental attribute - AT outcome. This was done (a) accounting for the sample size and quality scores of the articles (see previous section); (b) accounting only for the sample size score; (c) accounting only for the quality score; and (d) accounting for neither. The last three sets of computations represented sensitivity analyses determining the impact of study quality and sample size on the meta-analytic findings. When combinations of environmental attribute - AT outcome were examined by at least three studies using objective and at least three studies using perceived measures of an environmental attribute, supplementary summaries of findings by type of environmental measure (objective vs. perceived) were conducted (NB: three is the typical number of studies in meta-analyses included in Cohrane's database of systematic reviews) [43]. In line with methodological recommendations for meta-analyses [44], specific combinations of environmental attributes and AT outcomes examined less than five times were considered to be insufficiently studied.

To conduct the meta-analysis, positive associations were assigned a z-value of 1.96 (just significant at a $p$-level of 0.05), negative associations were given a $\mathrm{z}$ value of -1.96 and statistically non-significant associations a $z$-value of 0 . We did not use the exact $z$-values reported in the articles because the outcomes and measures were too diverse to allow the conduct of a traditional metaanalysis with accurate estimation and averaging of effect sizes. Given that, in the majority of cases, our approach underestimated the strength of associations by assigning to each significant finding the minimal z-value, the conclusions reported in this review are conservative. For each combination of environmental attribute - AT outcome variables, we computed a summary two-tailed $p$-value using Rosenthal's approach [45], whereby a summary weighted $z$-value and the two-tailed probability value associated with it were obtained. As noted earlier, weights incorporated the sum of the article sample size and quality scores, which were then multiplied by the fractional weight of the z-score described above. For the three sets of sensitivity analyses, we used respectively sample size scores, quality scores and a value of 1 (i.e., meaning no weighting) as 
weights. The following formula was used to obtain a summary weighted z-value:

$$
\text { Weighted } Z=\frac{\sum \text { weight }_{j} z_{j}}{\sqrt{\sum \text { weight }_{j}^{2}}}
$$

where ' $j$ ' stands for finding ' $\mathfrak{j}$ '.

Following Bland's recommendations [46], two-tailed $p$-values $<0.01$ were deemed to provide strong and $p$-values $<0.001$ very strong evidence of associations. A detailed example of how $\mathrm{p}$-values were derived is given in the supplementary material (Additional file 3).

\section{Results}

\section{Characteristics of selected articles}

We screened 19,005 references and examined the full text of 530. Forty-two articles met the selection criteria for this review (Fig. 1), all reporting findings from crosssectional studies (Table 1). Twenty-one studies were represented by a single article, while the remaining six studies had two to six articles selected for this review. Most articles reported findings from North America, followed by Europe, Asia, Oceania and South America. Africa was represented by a single article based on a small-scale, pilot study [47]. Over $80 \%$ of articles focused on older adults living in urban settings, while only five also included participants from suburban or rural areas [25, 35, 48-50].

Sample sizes ranged from 44 to 48,879 [25, 47], with eight (19.1\%) articles reporting data on less than 300 participants (considered to be a small sample size in this research field) [47, 48, 50-55]. Less than $30 \%$ of articles provided sufficient evidence for the sample representativeness (Table 2). Most studies (71.4\% articles; Table 2) recruited participants based on some type of stratification by environmental or socio-demographic characteristics. Fifteen articles from five studies recruited neighbourhoods stratified by both area-level socio-economic status (SES) and walkability $[4,6,34,51,56]$. Neighbourhoods were operationalised in a variety of ways, the most frequent of which were administrative/census areas (35.7\%), 400$500 \mathrm{~m}$ buffers around participants' residential addresses (21.4\%) and a participant-perceived area within a $10-20$ min walk from home (31.0\%).

AT was gauged using valid self-report measures in $76.2 \%$ of the cases (Table 2). Most articles (83.3\%) presented findings on environmental correlates of total walking for transport operationalised as a categorical, discrete or continuous outcome using a wide variety of questionnaires and different criteria of categorization/dichotomization. Only four articles, all from Hong Kong $[4,56-58]$, examined within-neighbourhood walking and two Belgian articles studied cycling for transport $[6,25]$.
Table 2 Summary of article quality assessment $(N=42)$

\begin{tabular}{|c|c|c|}
\hline Quality-assessment item [score] & $\begin{array}{l}\text { Number } \\
\text { of studies }\end{array}$ & $\%$ \\
\hline $\begin{array}{l}\text { 1. Study areas or participant recruitment stratified } \\
\text { by key environmental attributes [1] }\end{array}$ & 30 & 71.4 \\
\hline $\begin{array}{l}\text { 2. Response rate } \geq 60 \% \text { or sample representative } \\
\text { of the population [1] }\end{array}$ & 12 & 28.6 \\
\hline $\begin{array}{l}\text { 3. Active travel measures (outcomes) valid, or } \\
\text { well-established in the field [1] }\end{array}$ & 32 & 76.2 \\
\hline $\begin{array}{l}\text { 4. Analyses adjusted for key socio-demographic } \\
\text { covariates (at least age, sex and education } \\
\text { considered) [1] }\end{array}$ & 38 & 90.5 \\
\hline 5. Analyses adjusted for self-selection [1] & 4 & 9.5 \\
\hline $\begin{array}{l}\text { 6. Analytical approach - adjustment for } \\
\text { clustering (if needed) }[1 / 3]\end{array}$ & 30 & 71.4 \\
\hline $\begin{array}{l}\text { 7. Analytical approach - accounting for } \\
\text { distributional assumptions }[1 / 3]\end{array}$ & 36 & 85.7 \\
\hline $\begin{array}{l}\text { 8. Analytical approach - analyses conducted } \\
\text { and presented correctly }[1 / 3]\end{array}$ & 35 & 83.3 \\
\hline $\begin{array}{l}\text { 9. Did not (inappropriately) categorise continuous } \\
\text { environmental exposures [1] }\end{array}$ & 33 & 78.6 \\
\hline Total quality score [theoretical range: $0-7$ ], mean (SD) & 4.3 & 1.3 \\
\hline
\end{tabular}

Five studies across four continents used a measure of AT combining walking and cycling [32, 34, 47, 53, 59].

Access to/availability of services and destinations was the environmental category most frequently examined (78.6\% of articles), followed by pedestrian/cycling infrastructure and streetscape (59.5\%), and safety and traffic (57.1\%). Neighbourhood environmental attributes were more frequently gauged via self-reports than objective measures, with the exception of walkability which was exclusively assessed using objective Geographic Information Systems (GIS) data. Fifteen out of 23 articles reporting on self-report measures of the neighbourhood environment used one of the versions of the NEWS [29-31]. A variety of individual and environmental moderators of environmental correlates of AT were examined in 17 (40.5\%) articles (Table 1). The most frequently studied moderators were area-level income and age.

While most studies adjusted the analyses for key socio-demographic covariates, only a few considered residential self-selection (Table 2) [41, 54, 60, 61]. The adopted analytical approaches were adequate in the majority of cases, with non-adjustment for clustering and inappropriate categorisation of continuous environmental exposures being the most common problems. Detailed characteristics of each article, including quality assessment, are reported in Additional files 1 and 4.

Below, we present a detailed overview of the results separately for each AT outcome. Table 3 summarises the overall associations of environmental correlates with AT outcomes, while Table 4 summarises associations stratified by type (objective or perceived) of environmental 


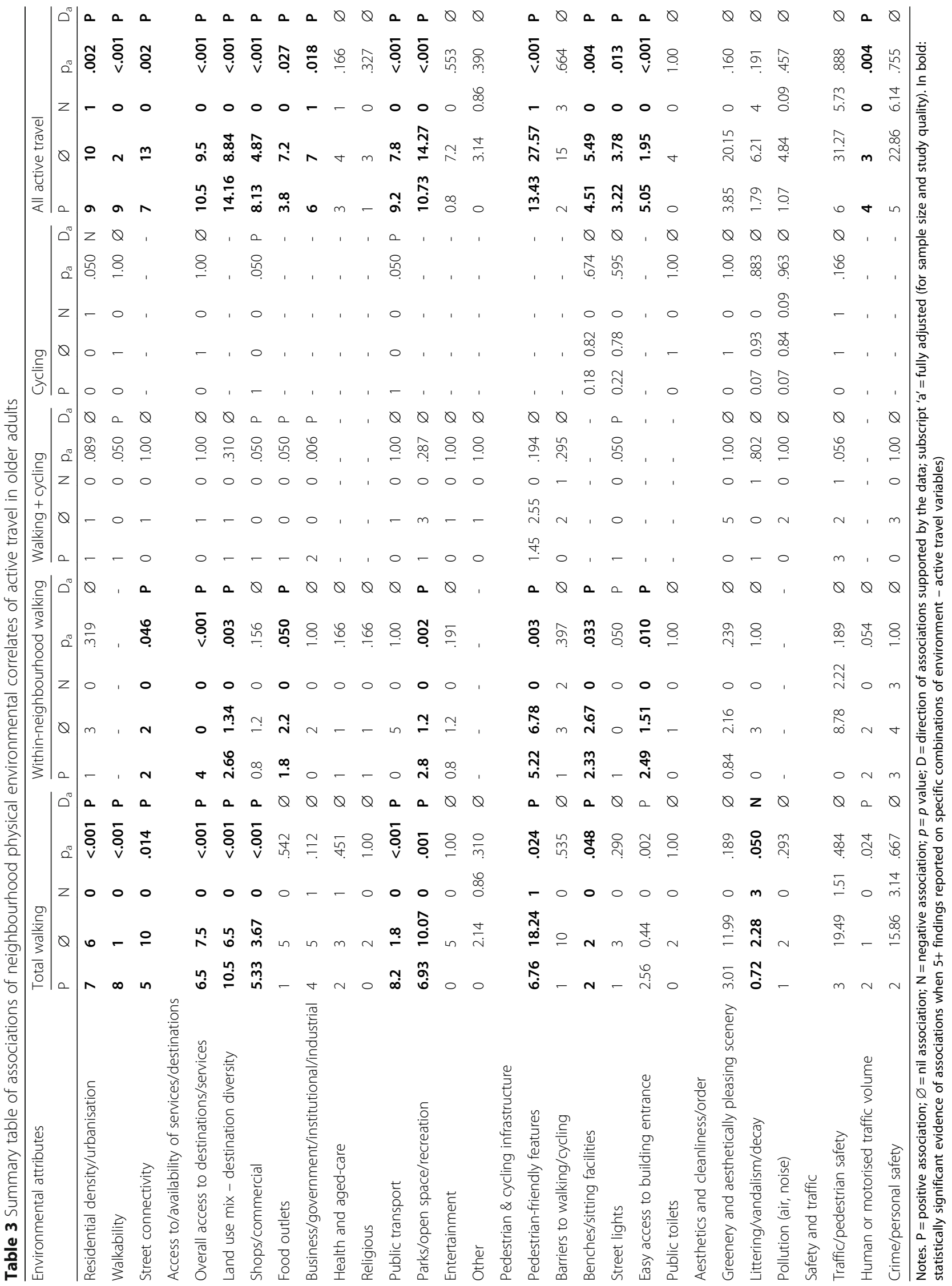


Table 4 Summary table of associations of neighbourhood physical environmental correlates with active travel in older adults by type of environmental measure (objective vs. perceived)

\begin{tabular}{|c|c|c|c|c|c|c|c|c|c|c|}
\hline \multirow[t]{2}{*}{ Environmental attributes } & \multicolumn{5}{|c|}{ Total walking } & \multicolumn{5}{|c|}{ All active travel } \\
\hline & $P$ & $\varnothing$ & $\mathrm{N}$ & $p_{a}$ & $\mathrm{D}_{\mathrm{a}}$ & $P$ & $\varnothing$ & $\mathrm{N}$ & $p_{a}$ & $D_{e}$ \\
\hline Residential density/urbanisation & 7 & 6 & 0 & $<.001$ & $P$ & 9 & 10 & 1 & .002 & $P$ \\
\hline Objective & 2 & 1 & 0 & .031 & $P$ & 3 & 1 & 1 & .145 & $\varnothing$ \\
\hline Perceived & 5 & 5 & 0 & .004 & $P$ & 6 & 9 & 0 & .006 & $\mathbf{P}$ \\
\hline Street connectivity & 5 & 10 & 0 & .014 & $P$ & 7 & 13 & 0 & .002 & $P$ \\
\hline Objective & 2 & 2 & 0 & .041 & $\mathbf{P}$ & 2 & 2 & 0 & .041 & $P$ \\
\hline Perceived & 3 & 8 & 0 & .113 & $\varnothing$ & 5 & 11 & 0 & .014 & $P$ \\
\hline \multicolumn{11}{|l|}{ Access to/availability of services/destinations } \\
\hline Land use mix - destination diversity & 10.5 & 6.5 & 0 & $<.001$ & $P$ & 14.16 & 8.84 & 0 & $<.001$ & $P$ \\
\hline Objective & 2.5 & 3.5 & 0 & .051 & $\varnothing$ & 2.5 & 3.5 & 0 & .051 & $\varnothing$ \\
\hline Perceived & 8 & 3 & 0 & $<.001$ & $\mathbf{P}$ & 11.66 & 5.34 & 0 & $<.001$ & $\mathbf{P}$ \\
\hline Public transport & 8.2 & 1.8 & 0 & $<.001$ & $P$ & 9.2 & 7.8 & 0 & $<.001$ & $P$ \\
\hline Objective & 4.2 & 0.8 & 0 & $<.001$ & $P$ & 4.2 & 2.8 & 0 & .002 & $P$ \\
\hline Perceived & 4 & 1 & 0 & $<.001$ & $P$ & 5 & 5 & 0 & .002 & $P$ \\
\hline Parks/open space/recreation & 6.93 & 10.07 & 0 & .001 & $P$ & 10.73 & 14.27 & 0 & $<.001$ & $P$ \\
\hline Objective & 2 & 8 & 0 & .229 & $\varnothing$ & 2.8 & 11.2 & 0 & .130 & $\varnothing$ \\
\hline Perceived & 4.93 & 2.07 & 0 & $<.001$ & $\mathbf{P}$ & 7.93 & 3.07 & 0 & $<.001$ & $\mathbf{P}$ \\
\hline \multicolumn{11}{|l|}{ Pedestrian \& cycling infrastructure } \\
\hline Pedestrian-friendly features & 6.76 & 18.24 & 1 & .024 & $P$ & 13.43 & 27.57 & 1 & $<.001$ & $P$ \\
\hline Objective & 2.25 & 5.75 & 0 & .090 & $\varnothing$ & 3.25 & 7.75 & 0 & .044 & $P$ \\
\hline Perceived & 4.51 & 12.49 & 1 & .114 & $\varnothing$ & 10.18 & 19.82 & 0 & $<.001$ & $P$ \\
\hline \multicolumn{11}{|l|}{ Aesthetics and cleanliness/order } \\
\hline Greenery and aesthetically pleasing scenery & 3.01 & 11.99 & 0 & .189 & $\varnothing$ & 3.85 & 20.15 & 0 & .160 & $\varnothing$ \\
\hline Objective & 0.5 & 2.50 & 0 & .569 & $\varnothing$ & 0.5 & 5.50 & 0 & .723 & $\varnothing$ \\
\hline Perceived & 2.51 & 9.49 & 0 & .237 & $\varnothing$ & 3.35 & 14.65 & 0 & .154 & $\varnothing$ \\
\hline \multicolumn{11}{|l|}{ Safety and traffic } \\
\hline Traffic/pedestrian safety & 3 & 19.49 & 1.51 & .484 & $\varnothing$ & 6 & 31.27 & 5.73 & .888 & $\varnothing$ \\
\hline Objective & 1 & 3 & 1 & 1.00 & $\varnothing$ & 4 & 4 & 1 & .043 & $\mathbf{P}$ \\
\hline Perceived & 2 & 16.49 & 0.51 & .432 & $\varnothing$ & 2 & 27.27 & 4.73 & .398 & $\varnothing$ \\
\hline
\end{tabular}

Notes. $\mathrm{P}=$ positive association; $\varnothing=$ nil association; $\mathrm{N}=$ negative association; $p=p$ value; $\mathrm{D}=$ direction of associations supported by the data; subscript 'a' $=$ fully adjusted (for sample size and study quality). Differences in associations between objective and perceived measures of environmental attributes are bolded

measure for combinations of environmental attributes and AT outcomes with at least three findings per type of environmental measure. Finally, Table 5 reports moderators of environmental correlates of AT.

\section{Neighbourhood environmental correlates of total walking for transport}

After accounting for sample size and article quality, strong evidence $(p<.01)$ of positive associations with total walking for transport was found for residential density/urbanisation, walkability, easy access to building entrance and several measures of access to/availability of services/destinations. The latter included overall access to destinations/services, land use mix - destination diversity, shops/commercial destinations, parks/open spaces/recreational destinations and public transport. Weaker evidence ( $p=0.014$ to 0.050$)$ of positive associations was found for aspects of pedestrian infrastructure (pedestrian-friendly features; benches/sitting facilities), street connectivity, traffic volume and lack of littering/ vandalism/decay. No sufficient evidence of associations was found for the remaining 13 environmental attributes, eight of which were examined five to 24 times. Among the attributes sufficiently examined (i.e., with five or more findings), the most consistent patterns of positive associations were observed for walkability, public transport, land use mix - destination diversity, shops commercial destinations, while the most consistent 
Table 5 Overview of moderators of neighbourhood physical environmental correlates of active travel in older adults

\begin{tabular}{|c|c|}
\hline Moderators & Environmental attribute (E) - AT outcome (AT) \\
\hline \multicolumn{2}{|c|}{ Individual: socio-demographics (self-reported) } \\
\hline $\begin{array}{l}\text { Age (Barnes et al., in } \\
\text { press) [62] }\end{array}$ & $\begin{array}{l}\text { E: (1) Walkability; (2) Public transport } \\
\text { AT: (1) Total walking }\end{array}$ \\
\hline $\begin{array}{l}\text { Age (Shigematsu et } \\
\text { al., 2009) [63] }\end{array}$ & $\begin{array}{l}\text { E: (1) Residential density; (2) Access to destinations/ } \\
\text { services; (3) Land use mix - destination diversity; } \\
\text { (4) Street connectivity; (5) Pedestrian-friendly } \\
\text { features; (6) Greenery and aesthetically pleasing } \\
\text { scenery; (7) Traffic/pedestrian safety; (8) Public } \\
\text { transport; (9) Crime/personal safety; (10) Parks/ } \\
\text { open space/recreation destinations } \\
\text { AT: (1) Total walking }\end{array}$ \\
\hline
\end{tabular}

Sex (Inoue et al., 2011) [35]

\section{E: (1) Residential density; (2) Shops/commercial} destinations; (3) Public transport; (4) Pedestrianfriendly features; (5) Traffic/pedestrian safety; (6) Crime/personal safety; (7) Park/open space/recreation; (8) Greenery and aesthetically pleasing scenery AT: (1) Total walking

Age
Sex
Education (Cerin et al
2014) [58]

Age

Sex (Van

Cauwenberg et al., 2012) [25]

Living arrangements (Tsai et al., 2013) [64]

\section{E: (1) Residential density; (2) Access to destinations/} services; (3) Land use mix - destination diversity; (4) Street connectivity; (5) Pedestrian-friendly features; (6) Traffic/pedestrian safety; (7) Public transport; (8) Crime/personal safety; (9) Barriers to walking/cycling; (10) Easy access to building entrance; (11) Human or motorised traffic volume; (12) Benches/sitting facilities AT: (1) Total walking; (2) Within-neighbourhood walking

E: (1) Access to destinations/services; (2) Shops/commercial destinations; (3) Public transport; (4) Public toilets;

(5) Benches/sitting facilities; (6) Traffic/pedestrian safety;

(7) Pedestrian-friendly features; (8) Crime/personal safety;

(9) Street lights; (10) Littering/vandalism/decay; (11) Pollution;

(12) Greenery and aesthetically pleasing scenery

AT: (1) Total walking; (2) Cycling

E: (1) Traffic/pedestrian safety; (2) Barriers to walking/ cycling; (3) Easy access to building entrance;

(4) Land use mix - destination diversity

AT: (1) Total walking

Individual: psychosocial factors (perceived)

$\begin{array}{ll}\begin{array}{l}\text { Social support for } \\ \text { physical activity }\end{array} & \begin{array}{l}\text { E: (1) Walkability; (2) Parks/open space/recreation } \\ \text { destinations }\end{array} \\ \text { Self-efficacy for } & \text { AT: (1) Total walking }\end{array}$

physical activity

Perceived barriers to

physical activity

(Carlson et al., 2012)

[65]

Individual: vehicle ownership/driving status (self-reported)

Driving status

(Ding et al., 2014) [22]

E: (1) Residential density; (2) Access to destinations/ services; (3) Land use mix - destination diversity;

(4) Street connectivity; (5) Pedestrian-friendly features;

(6) Greenery and aesthetically pleasing scenery;

(7) Traffic/pedestrian safety; (8) Public transport;
- No significant moderating effects.

- Positive associations with (10) Parks/open space/recreation (park near home) only in $75+$ year olds.

- Positive associations with (2) Shops/commercial destinations and (7) Park/open space/recreation destinations only in women.

- Positive associations with (4) Pedestrian-friendly features and (8) Greenery and aesthetically pleasing scenery only in men.

- Negative associations with (5) Traffic/pedestrian safety and (6) Crime/personal safety only in men.

- Positive associations of (3) Land use mix destination diversity and (12) Benches/sitting facilities with (2) Within-neighbourhood walking only in $75+$ year-olds.

- Negative associations of (8) Crime/personal safety

and (2) Within-neighbourhood walking only in women.

- Positive associations with (10) Littering/vandalism/ decay and (1) Total walking in all but 75+ year-old women.

- Complex Age by Sex interaction on (11) Pollution (1)

Total walking associations. All associations positive. - Significant Age by Sex interaction on (4) Public toilets, (6) Traffic/pedestrian safety and (2) Cycling associations. However, no significant associations in subgroups (public toilets; presence of crossings) or all associations negative (traffic safety).

- Positive associations of (9) Street lights with (2) Cycling only in $<75$-year old women.

- See also interactions of Urbanism by Age or Sex below.

- Positive association with (3) Easy access to building entrance only in those living alone.

- Stronger associations with (1) Walkability in those with higher social support and self-efficacy, and lower perceived barriers to physical activity.
- No significant moderating effects. 
Table 5 Overview of moderators of neighbourhood physical environmental correlates of active travel in older adults (Continued)

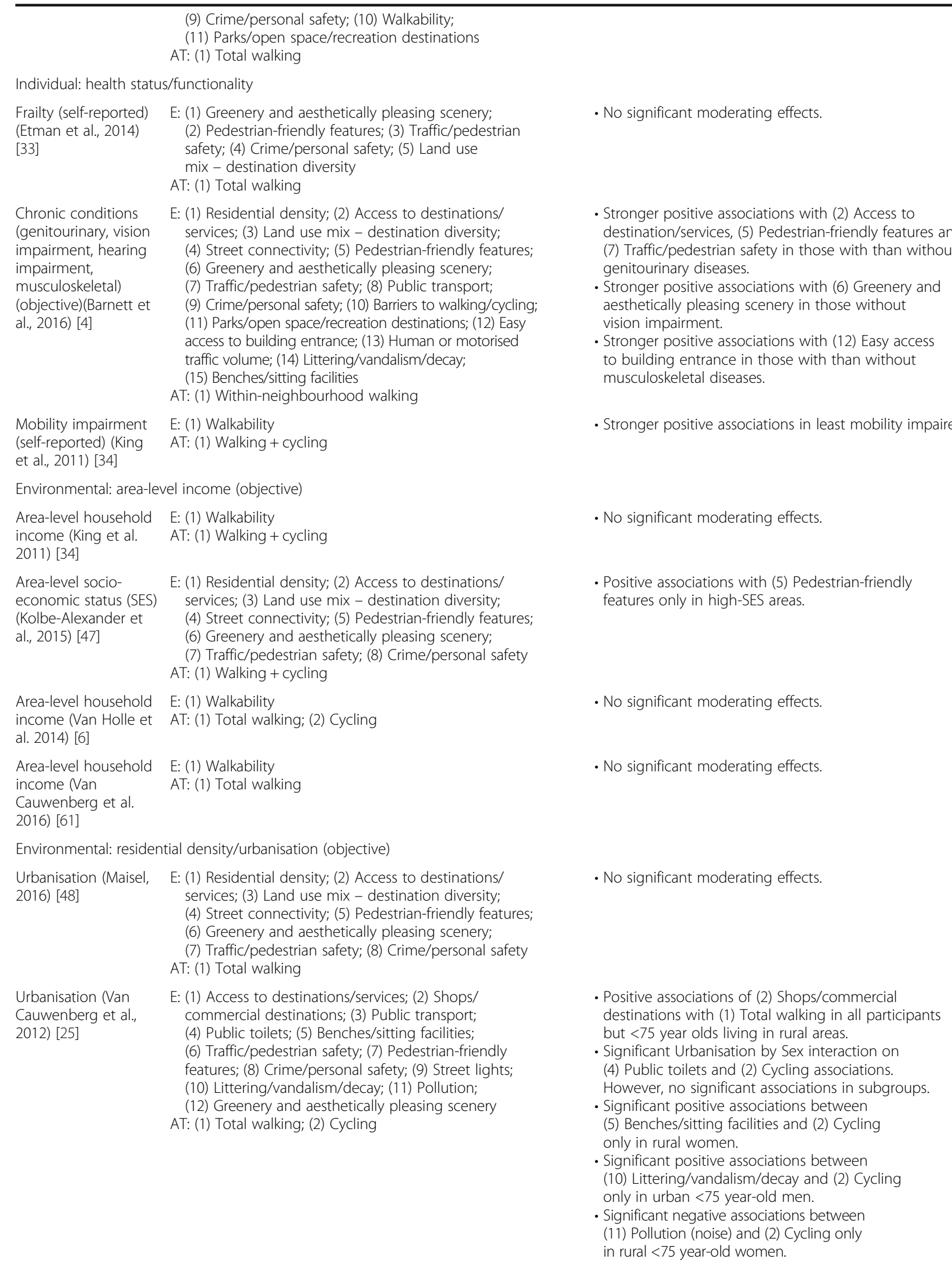


Table 5 Overview of moderators of neighbourhood physical environmental correlates of active travel in older adults (Continued)

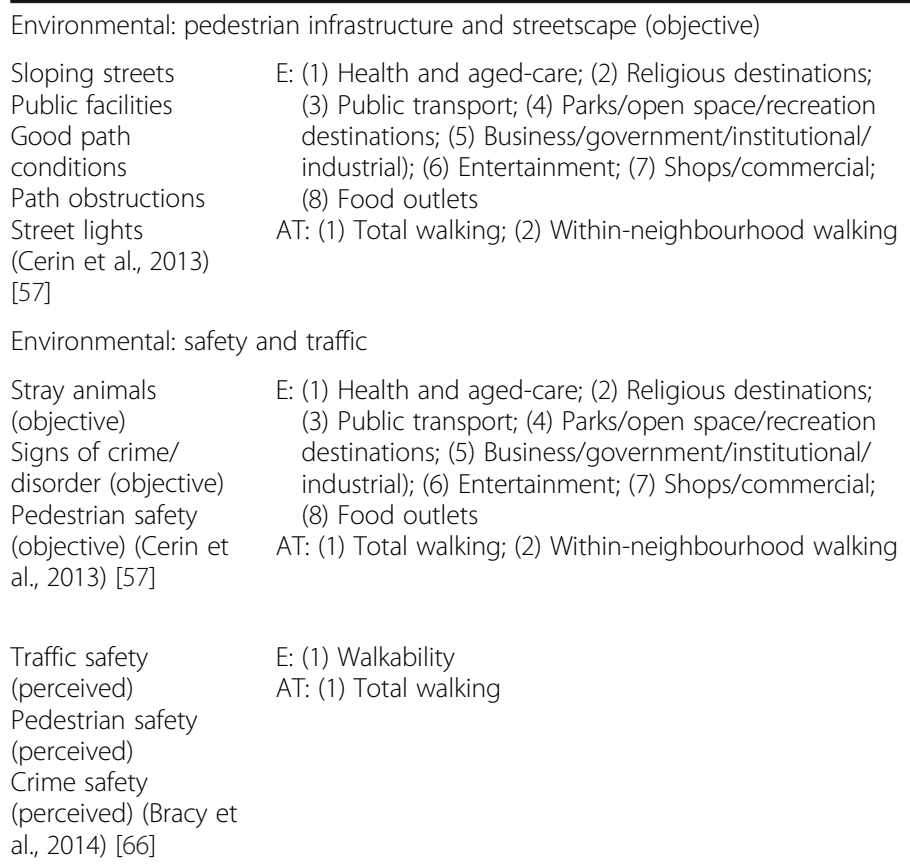

Sloping streets

Public facilities

Good path

conditions

Path obstructions

Street lights

(Cerin et al., 2013)

[57]
E: (1) Health and aged-care; (2) Religious destinations; (3) Public transport; (4) Parks/open space/recreation destinations; (5) Business/government/institutional/ industrial); (6) Entertainment; (7) Shops/commercial; (8) Food outlets
AT: (1) Total walking; (2) Within-neighbourhood walking

Environmental: safety and traffic

Stray animals

(objective)

Signs of crime/

disorder (objective)

Pedestrian safety

(objective) (Cerin et

al., 2013) [57]

Traffic safety

(perceived)

Pedestrian safety

(perceived)

Crime safety

(perceived) (Bracy et

al., 2014) [66]

\begin{abstract}
E: (1) Health and aged-care; (2) Religious destinations; (3) Public transport; (4) Parks/open space/recreation destinations; (5) Business/government/institutional/ industrial); (6) Entertainment; (7) Shops/commercial; (8) Food outlets

AT: (1) Total walking; (2) Within-neighbourhood walking
\end{abstract}

E: (1) Walkability

AT: (1) Total walking

- Stronger positive associations between

(7) Shops/commercial destinations and

(2) within-neighbourhood walking in areas

with more path obstructions and fewer sloping streets.

- Stronger positive associations between (7) Food outlets (shops and grocery stores) and (2) withinneighbourhood walking in areas with fewer path obstructions and no sloping streets.

- Stronger positive associations between (3) Public transport and (1) Total walking; and (4) Parks/open space/recreation destinations and (2) Within-neighbourhood walking in areas with fewer stray animals.

- Stronger positive associations of (4) Parks/open space/ recreation destinations and (6) Entertainment and (2) Within-neighbourhood walking in areas with fewer signs of crime/disorder.

- No significant moderating effects. patterns of nil associations were found for barriers to walking/cycling, entertainment and food outlets. With the exception of two environmental characteristics (benches/ sitting facilities and littering/vandalism/decay), the above conclusions held true in analyses unadjusted for sample size, article quality or both (Additional file 5).

The evidence of a positive association between street connectivity and total walking for transport was stronger for objective than perceived measures of street connectivity (Table 4). The opposite was true for land use mix destination diversity and parks/open space/recreation. No differences in patterns of associations with total walking for transport were observed between objective and perceived measures of residential density/urbanisation, public transport, pedestrian-friendly features, greenery and aesthetically pleasing scenery and traffic/pedestrian safety.

\section{Moderators of associations}

Age was investigated as a moderator of associations between environmental attributes and total walking for transport in four different studies (Table 5) [25, 58, 62, 63]. Only a few significant moderating effects were observed, with stronger positive associations of parks with total walking for transport being reported in older participants (75+ year olds) in the USA [63]. In contrast, in a Belgian study, younger participants ( $<75$ year olds) showed stronger associations with littering/vandalism/decay and shops/ commercial destinations [25]. Sex was examined as a moderator in Japanese [35], Hong Kong [58] and Belgian samples [25]. While sex did not moderate the effects of the neighbourhood environment on total walking in Hong Kong older adults [58], weaker associations were found with a measure of littering/vandalism/decay among older women than other socio-demographic subgroups in Belgium [25]. Access to shops and exercise facilities were found to be more strongly positively associated with total walking for transport in Japanese women than men, while men showed stronger associations with aspects related to safety, aesthetics and pedestrian infrastructure [35]. Other socio-demographic moderators (education and living arrangement) were only examined in single studies [58, 64], as were psychosocial factors [65], driving status [22], and frailty [33].

Two articles from the same Belgian study estimated the moderating effect of area-level income on walkability and total walking for transport and found none $[6,61]$. Two articles also examined level of urbanisation as a moderator of a wide range of perceived neighbourhood attributes and total walking for transport. No evidence was found in the USA [48] and very limited evidence in Belgium [25], where stronger associations between shops and walking were detected in more urban areas (Table 5). Using environmental measures derived from detailed neighbourhood audits, a study conducted in Hong Kong examined aspects of pedestrian infrastructure and safety as moderators of associations between access/availability of destinations and total walking for transport [57]. Only the presence of stray animals was found to modify the association between prevalence of public transport 
points and walking, whereby residents of neighbourhood with no or few stray animals showed stronger positive associations than their counterparts. No significant moderating effects of aspects of perceived safety on the associations between walkability and total walking for transport were found in a USA sample [66].

\section{Neighbourhood environmental correlates of within- neighbourhood walking for transport}

Only four articles from two Hong Kong studies examined neighbourhood environmental correlates of withinneighbourhood walking [4, 56-58]. Strong evidence was found for positive associations with overall access to destinations/services, land use mix - destination diversity, parks/open space/recreational destinations, pedestrianfriendly features and easy access to building entrance (Table 3). Weaker evidence of positive associations was detected for street connectivity, access to food outlets, street lights and having benches/sitting facilities in the neighbourhood. Insufficient evidence of associations was found for the remaining 14 environmental attributes studied, while walkability and pollution were not investigated. The patterns of relationships were largely independent of the type of adjustment used in the metaanalyses (Additional file 5).

\section{Moderators of associations}

Age, sex, education [58] and chronic conditions [4] were investigated as moderators of within-neighbourhood walking only in single studies (Table 5). Land use mix destination diversity and the presence of benches and sitting facilities were identified as significant correlates in older (75+ year olds) but not younger senior residents (65-74 year olds) [58]. Only one of 12 investigated moderating effects of sex was statistically significant and none were significant for education [58]. Having vs. not having a genitourinary or musculoskeletal disease was predictive of stronger associations with four out of 15 environmental attributes [4]. In contrast, not having vs. having visual impairment yielded stronger positive associations of neighbourhood aesthetics with weekly frequency and amounts of within-neighbourhood walking for transport.

\section{Neighbourhood environmental correlates of combined measures of walking and cycling for transport}

The most frequently examined environmental features among the five articles reporting on combined measures of walking and cycling were parks/open spaces/recreational destinations, pedestrian-friendly features, greenery and aesthetically pleasing scenery, and traffic/pedestrian safety (Table 3). Evidence of positive associations was found for business/government/institutional/industrial destinations, walkability, shops/commercial destinations, food outlets and street lights. However, only the first of these characteristics was examined in more than one article. Insufficient evidence of associations was reported for 15 other neighbourhood attributes of which only two were investigated five or more times (greenery and aesthetically pleasing scenery and traffic/pedestrian safety). With the exception of traffic/pedestrian safety, the patterns of relationships were independent of the type of adjustment used in the meta-analyses (Additional file 5). All positive associations of traffic/pedestrian safety with walking and cycling were found when using objective measures of this environmental attribute [32], while perceived traffic/pedestrian safety yielded nil [59] or unexpected associations [47].

\section{Moderators of associations}

Only two articles studied moderators of associations of environmental attributes with combined measures of walking and cycling for transport. Both of these articles examined the moderating effect of area-level SES (Table 5) [34, 47]. Area-level household income did not moderate the associations with objectively-assessed walkability in the USA [34]. However, in a South African pilot sample, perceived pedestrian-friendly features were positively associated with this AT outcome only in highSES areas [47]. Yet, the latter article did not conduct a formal analysis of moderators (Additional file 4).

\section{Neighbourhood environmental correlates of cycling for transport}

Only two Belgian studies examined neighbourhood environmental correlates of cycling for transport in older adults $[6,25]$. These studies examined a total of 12 environmental correlates (Table 3). A large population sample provided evidence for positive relations with access to public transport and shops/commercial services, and negative associations with urbanisation [25]. No differences in conclusions were observed when using different types of adjustment (Additional file 5).

\section{Moderators of associations}

A study examined area-level-household income as a moderator of the effects of walkability and found insufficient evidence of moderation (Table 5) [6]. Another large-scale Belgian study estimated the moderating effects of age, sex and urbanisation level on the associations of 12 perceived environmental attributes and cycling or not cycling for transport on a daily basis [25]. Complex age by sex, age by urbanisation and age by sex by urbanisation interaction effects were observed (detailed in Table 5). 


\section{Neighbourhood environmental correlates of active travel (all outcomes combined)}

The last set of columns in Table 3 provides a summary of the evidence of environmental correlates of all AT outcomes combined. Most of the evidence mirrored that of total walking for transport, which was the most frequently examined AT outcome. Differences were observed only with regards to four environmental characteristics. These were food outlets, business/government/ institutional/industrial destinations, street lights and littering/vandalism/decay, whereby positive associations were found between the first three categories of environmental attributes with all AT outcomes but not with total walking for transport. The opposite was true for littering/vandalism/decay. Different types of adjustment for quality and sample size did not yield different conclusions (Additional file 5).

Different patterns of associations were found between objective and perceived measures of four environmental attributes and all AT outcomes (Table 4). Only perceived measures of residential density/urbanisation, land use mix - destination diversity and parks/open space/recreation destinations provided sufficient evidence of positive associations. The opposite held true for traffic/pedestrian safety.

\section{Discussion}

In the last decade, the health, transportation and environmental sustainability sectors have become increasingly interested in finding ways to promote AT in older adults via environmental and policy interventions [10, 67-70]. This interest has been accompanied by an eight-fold increase in the number of articles on neighbourhood physical environmental correlates of older adults' AT since the latest systematic review on this topic was published [19]. We have critically reviewed the empirical evidence and developed a novel meta-analytic procedure to statistically summarise and combine the evidence published in this millennium.

\section{Neighbourhood walkability and its components}

We found very strong and consistent support for a positive association between objectively-assessed neighbourhood walkability and older adults' total walking for transport. This relationship applied to older adults living in high- as well as low-income neighbourhoods $[6,34,61]$. Moderate-to-strong evidence of positive associations with total walking for transport also emerged for all individual components of walkability - namely, residential density, street connectivity and land use mix. As these three neighbourhood attributes have been also identified as independent contributors to walking for transport in younger adults (aged 18-65 years) from 14 cities across the globe [71], these findings speak in favour of the universal importance of walkability as a determinant of walking for transport across adulthood.

Among the various aspects of walkability, land use mix and access to destinations were more consistent correlates of older adults' AT than residential density and street connectivity. This is understandable given that easy access to a variety of destinations is an essential component of AT, while residential density is usually a proxy for availability of destinations and services, and street connectivity is considered a facilitator rather than an essential factor for AT [72, 73]. Moreover, the effects of residential density may differ by AT outcome and geographical context. For example, a large populationbased Belgian study reported a negative association between urbanisation level and older adults' cycling for transport [25], which is in line with a multi-country study on adults aged 18-64 years [24]. Denser areas are typified by short distances to destinations and higher levels of traffic volume and hazards, possibly making cycling a less safe and less convenient mode of transport than walking.

We found no support for an association between residential density and within-neighbourhood walking. This may be attributable to all the evidence originating from an ultra-dense city (Hong Kong) [4, 58]. Increasing density in already highly dense areas may result in a decrease in walking for transport because distances to commercial, transportation and other services become very short and/or people may opt to 'chain' multiple trips to a single trip. In this regard, a multi-country study identified thresholds of 7,500-12,000 dwellings/ $\mathrm{km}^{2}$ for objectively-assessed residential density after which the odds and frequency of adults' walking for transport plateaued or decreased [71]. It is unknown whether these threshold values also apply to older adults. We need multi-country studies with comparable methodologies that would allow data pooling to be able to examine threshold effects and identity the optimal amount of density that supports walking and cycling for transport in older adults.

\section{Destinations that matter to older adults}

While the availability of destinations is undoubtedly a crucial determinant of AT, it is plausible to assume that not all types of destinations are equally important to older adults [54, 57]. Among the nine categories of destinations examined in this review, public transport stops, shops/commercial destinations and parks/open spaces/ recreational facilities were the most consistent correlates of AT, with most of the evidence coming from studies examining total walking for transport. Some, but weaker, evidence was also found for availability of food outlets and various business/institutional/industrial destinations with respect to all AT outcomes. In agreement with 
these findings, several travel diary studies revealed that shopping (including food purchases), errands, recreational activities and social activities were the most frequently reported purposes of all-mode and/or AT trips among older adults in Australia [74], Hong Kong [75] and the UK [53]. Also, shops, services, food outlets, transit stops and meeting points emerged as the most frequently visited destinations by Canadian [54] and Hong Kong older adults [75]. Good access to public transport is particularly important to older adults who do not live in destination-rich neighbourhoods [57] and have limited or no access to private travel options $[22,76]$.

\section{Pedestrian infrastructure and streetscape}

We identified several important aspects of pedestrian infrastructure and streetscape that may promote AT in older adults. Pedestrian-friendly features, such as the presence of well-maintained footpaths and indoor places for walking, were found to be positively related to total as well as within-neighbourhood walking. The same held true for availability of benches/sitting facilities and having easy access to the residential building entrance, while the presence of street lights was associated with more $\mathrm{AT}$ in general. Overall, these findings mirror those of a review of qualitative investigations [77] and a recent experimental study [78] reporting that older adults considered the presence, quality and pedestrian-friendliness of footpaths as the most important set of microenvironmental features encouraging walking.

Older adults typically experience increasing levels of mobility limitations and fear of falling due to chronic health conditions, such as musculoskeletal diseases [79] and sensory impairments [80]. For this reason, they are more vulnerable to physically challenging environments than younger individuals. Being able to easily get out of one's home into a safe, pedestrianfriendly environment that provides opportunities for resting (benches/sitting facilities) may be decisive factors for older adults' engaging in AT [64]. While this review and meta-analysis support these contentions, it should be noted that, with the exception of pedestrian-friendly features, very few quantitative studies examined the effects of the above neighbourhood attributes. Also, findings on pedestrian-friendly features and total walking for transport were more heterogeneous than those related to walkability and its components. While availability of destinations is an essential determinant of AT, the importance of walking infrastructure for AT is likely to be dependent on the presence of relevant destinations and one's functional mobility. The only study that examined the moderating effects of diagnosed chronic conditions affecting functional mobility on the associations of pedestrian infrastructure with walking for transport found stronger positive relationships in those with genitourinary and musculoskeletal diseases than in those without these diseases [4]. Moreover, the presence of benches/sitting facilities was related to within-neighbourhood walking for transport only in older Hong Kong elders (aged 75+ years) [58]. Finally, in an experimental study on environmental features supporting AT in Belgian older adults, Van Cauwenberg and colleagues observed that the availability of benches/sitting facilities was particularly important for those with functional mobility limitations and fear of falling [78].

\section{Other neighbourhood built environment characteristics}

No evidence of associations with AT was found for most variables measuring neighbourhood aesthetics, cleanliness/order, traffic and crime-related safety. Weak evidence of a negative association was observed between the presence of litter, vandalism and decay and total walking for transport, suggesting that sometimes signs of neighbourhood disorder may discourage walking in older adults. Also, higher human and motorized traffic volumes tended to be positively correlated with walking for transport likely due to destination-rich, walkable areas also being heavily trafficked and visited by a large number of people. Some of these findings are in contrast to those reported in qualitative studies which emphasise the importance of safety [77]. The lack of consistent quantitative evidence of an effect of aesthetics and safety on AT in older adults mirrors the findings observed in younger adults [24, 81, 82]. There are several potential reasons responsible for these findings. It has been suggested that some individuals may engage in AT regardless of aesthetic or safety issues because they have no other option (e.g., they do not have access to public transport or own a car) [24, 76]. Measures of aesthetics and safety are typically generic and based on subjective evaluations that have different meaning and behavioural implications for different people [83, 84]. Neighbourhood aesthetics and safety may represent peripheral facilitators rather than essential determinants of AT and, for example, moderate the effects of access to destinations and services as observed in a study on Hong Kong older adults [57]. In this regard, Alfonzo hypothesised that the need for safety and aesthetics could influence walking only if the more important need of access to services is met [73]. Finally, most studies were conducted in high-income countries in which safety may not really be an issue, especially during the day when most of the AT is performed. In this respect, a multi-country study of adults including high- as well as mid-income, less safe countries (e.g., Mexico and Colombia) revealed that the positive association of safety from crime with PA was much stronger at the betweencountry than within-country level [85]. 


\section{Theoretical and methodological considerations}

Research in the area of environmental correlates of AT has been inspired by Sallis et al.'s socio-ecological model of active living [23]. According to this model, physical environmental factors shape AT in a contextspecific manner. Because walking and cycling for transport have different needs, they are likely influenced by different environmental features. Context specificity has also a geographical dimension according to which neighbourhood environmental attributes are hypothesised to impact on AT within and outside the neighbourhood in different ways [86, 87]. Yet, an analysis of the current evidence reveals that most studies focused on total walking for transport, irrespective of the location where it occurred. Also, more studies examined cycling in combination with walking than as a distinct AT outcome. Although from a public health perspective it is undoubtedly important to identify neighbourhood characteristics that are associated with higher overall levels of activity (e.g., total walking or total AT), from a behavioural perspective it is essential to understand how these characteristics impact on specific behaviours as this knowledge can more effectively guide environmental interventions.

Beside context specificity, another key tenet of the socio-ecological model of active living is the presence of interactions between factors shaping physical activity behaviour [23]. The effect of an environmental characteristic on AT is hypothesised to depend on (i.e., be moderated by) individual, social and other environmental factors. Human behaviour is a complex phenomenon that can seldom be accurately explained by simple additive models of exposures and individual characteristics. Yet, this review has revealed a dearth of findings on interactions. Eight of 11 studies that examined individuallevel moderators of environment-AT associations found significant interaction effects of socio-demographic, health or psychosocial factors. Among the seven studies that investigated environmental moderators, three observed significant effects. Collectively, these findings suggest that different groups of older adults are likely to require different types of environmental interventions and the effectiveness of certain environmental modifications (e.g., increased access to public transport) may depend on other environmental conditions being met (e.g., high-quality pedestrian infrastructure). Solid knowledge of these moderating factors is necessary to effectively target the most vulnerable population subgroups of older adults and maximise the impact of environmental interventions. Whenever possible, this line of research needs to undertake theory-driven rather than purely exploratory analyses, given the large number of testable interactions effects arising from studies of AT adopting an ecological approach.
The presence of unexplored interaction effects may be one of the reasons for the heterogeneous findings observed in this review with respect to several categories of environmental factors. Another potential source of heterogeneity may be the different types of exposure measures. Self-report measures of land use mix and access to recreational destinations yielded stronger evidence of positive associations than did objective measures. The opposite was true for traffic/pedestrian safety with respect to $\mathrm{AT}$, and for street connectivity with respect to walking for transport. Older adults who engage in AT are likely to be more aware of the availability of destinations within their neighbourhood than those who do not walk or cycle for transport. They are certainly more aware of the destinations that they visit (e.g., specific type of shop or food outlet). In contrast, objective measures of land use mix - destination diversity and destination availability may not be optimally operationalised and include a substantial number of places, services and/or land uses of little importance to older adults. In fact, not much is known about the optimal mix and number of destination types that might promote AT in this age group. Also, objective measures of destination availability are typically obtained for whole administrative areas [88-91] or home-centred buffers of various sizes [33, 92, 93], while self-report measures usually define a neighbourhood as an area within 10-20 min walk from a participant's home [58, 94, 95]. Given that there is large inter-individual variability in functional mobility and walking speed among older adults [96], defining a neighbourhood using time as a parameter (as in self-report measures) may be more appropriate for this age group than defining it in terms of distance (as in objective measures of the environment). This could in part explain why this review found that objective distance-based measures of destination availability performed worse as correlates of AT than their self-report counterparts. It is interesting that, in contrast, objectively-assessed street connectivity and traffic/pedestrian safety were more consistently associated to AT outcomes than self-report measures of the same. It is possible that these neighbourhood attributes are more susceptible to individual response biases or more difficult to recall than destination availability due to them not being the main focus of attention during AT.

Apart from various methodological issues (e.g., confounding, insufficient statistical power, use of measures with varying metric characteristics), some of the heterogeneity in findings observed in this review may be also the result of limited within-study and large betweenstudy variability in exposures. For example, as noted earlier, there is evidence that residential density may be curvilinearly related to walking for transport in adults $[24,71]$. However, the predominately positive concavedownward relationship became apparent only after 
pooling data from different cities varying in residential density. This shape of relationship implies that data collected in low-to-mid density (e.g., $<15,000$ dwellings $\mathrm{km}^{2}$ [71]) areas may show a positive association between residential density and walking for transport, while studies conducted in high density areas (e.g., >15,000 [71] or $>30,000$ [58]) may yield nil or negative associations. In this review, we found this to be the case for studies based in the USA [22], Australia [97], eastern European countries [95], Belgium (low-to-medium density areas) [25], and Japan [35] and Hong Kong [58] (high density areas). To resolve discrepant findings potentially due to restricted variability in exposures and the presence of non-linear dose-repose relationships, pooled analyses of comparable data from geographical locations varying in exposure are necessary.

\section{Weaknesses of available evidence}

One of the main weaknesses of the available evidence on physical environmental correlates of older adults' AT is its cross-sectional nature. Cross-sectional studies cannot establish causality because they are affected by a range of threats to validity, residential self-selection being one of the most important even in good quality studies [98]. Only four of 42 reviewed articles attempted to address self-selection by including reasons for living in a specific neighbourhood or enjoyment of an active lifestyle as covariates in regression analyses $[41,54,60,61]$. All four studies found evidence of positive associations between aspects of walkability and AT in older adults, indicating that the effects are unlikely to be entirely due to residential self-selection. Other reviews and studies that examined the potential effect of residential self-selection on PA [99] or related health outcomes [100] in other age groups reported similar patterns of findings and, in some instances, observed stronger associations in the expected direction after adjustment for self-selection [101]. Apart from more good-quality cross-sectional studies, this research field would benefit from stronger causal evidence based on well-conducted prospective and quasi-experimental studies, which may be challenging as the amount and rate of environmental changes is often insufficient during the life of a typical study to significantly impact on AT [99]. Prospective studies examining the effects of post-retirement relocation to less or more activity-friendly neighbourhoods on AT might address the likely lack of variability in environmental changes encountered in prospective studies of non-movers. However, studies of movers need to account for the fact that people who relocate to a new neighbourhood need a certain amount of time to become part of the community and familiarise with its physical environment. Also, prospective studies of movers raise selection bias concerns since movers may differ from non-movers in important ways that impact on their AT and adaptation to new environments.

Sampling bias was identified as a significant threat to validity in over $70 \%$ of the reviewed articles, with many studies reporting very low $[22,32,34,50,53,54,63,65,66]$ or no information on response rates $[47,48,50-52,55,64$, $70,89,93,95,97]$. Optimally, studies should not only report response rates but also provide an assessment of the representativeness of the sample and describe the implications of an identified or potential pattern of selection bias. It is particularly important to implement strategies aimed at maximising the response rate and participant retention. These have been extensively examined [102] and successfully employed in studies on neighbourhood correlates of AT in midaged adults [103]. Mailed surveys and face-to-face interviews appear to be associated with better response rates than web-based surveys and phone interviews in older adults [104].

Although most of the reviewed articles reported using validated measures of AT, they were all based on selfreports. AT is a form of incidental PA that may be more difficult to recall than leisure-time PA [86]. The use of Geographic Positioning System (GPS) devices with automated algorithms able to identify AT trips and modes (cycling versus walking) would help reduce measurement error and also clarify the extent to which neighbourhood environmental attributes impact on the geographical context of AT (e.g., walking within and outside the neighbourhood) $[105,106]$.

The analytical approaches adopted in the reviewed articles were often acceptable. Among the unacceptable practices were: (1) the failure to account for area-level clustering invalidating the standard errors of regression coefficients and, thus, conclusions [39]; (2) the failure to report exact p-values, standard errors or $95 \%$ confidence intervals of main effects; (3) the failure to probe significant interaction effects by estimating regression coefficients and their standard errors at meaningful values of the moderator; and (4) the tendency to transform continuous outcomes and exposures into categories. The latter issue has been shown not only to lead to loss of statistical power but also to increase residual confounding and the likelihood of spurious associations and interaction effects [40].

\section{Strengths and weaknesses of systematic review}

There are several strengths to this systematic review and meta-analysis. We devised a conservative meta-analytic approach that allowed a quantitative synthesis of findings that did not rely on the here-inappropriate use 
of precise effect sizes. Both published peer-reviewed scientific and grey literatures were examined to address possible article selection biases. When possible, findings were presented by AT mode and environmental measure type (objective and self-reported). Quality assessment and sample size information was incorporated in the meta-analyses and sensitivity analyses were conducted to examine the effects of this information on the conclusions. Limitations include the inability to more accurately quantify associations due to large variability in exposures and outcomes; in some instances, not accounting for correlated findings extracted from the same study (e.g., multiple outcomes falling into the same AT category); the inability to examine the moderating effects of neighbourhood size on the strength of environment AT associations due to studies not examining this issue (except for Etman et al.'s study, with no clear emerging patterns of associations [33]); and the exclusive focus on work published in English which possibly led to an overrepresentation of studies from developed countries.

\section{Unanswered questions and future research}

Many substantive questions on the topic of this review remain unanswered. To guide future research, Fig. 2 depicts a proposed conceptual model that focuses on the most important neighbourhood feature for AT: destinations. Individuals are more likely to walk or cycle to/ from places if they are within affordable distance from home. Yet, this review suggests that not all destinations are relevant to older adults. Shops, food outlets, commercial and government services, public transport stops and recreational facilities appear to be particularly important. However, little is known about the mix and density of types of shops, services and facilities that are necessary to optimally promote AT among older adults.

An optimal mix and density of destinations and services catering for older adults' daily needs may be available in the community. However, whether older residents will wish to visit them depends on their appeal. Further, residents will walk or cycle to 'appealing' destinations and services only if they are sufficiently accessible by $\mathrm{AT}$, and $\mathrm{AT}$ is a more appealing option than motorised transport. Figure 2 lists potential individual and environmental factors influencing access to destinations and the appeal of destinations and AT. These 'accessibility' and 'appeal' factors are hypothesised to moderate the associations of destination types and mixes with AT by determining one's capacity to reach a destination by AT ('accessibility'), one's desire or need to visit a destination (destination 'appeal'), and one's preference to use AT (AT 'appeal'). These factors are also postulated to independently influence AT, but only when destinations are available [73]. These propositions are in line with the moderating effects of sex [25, 35], age $[25,58,63]$, health status $[4,34]$, pedestrian infrastructure and pedestrian/crime safety [57] found in a few studies included in this review, and are based on concepts from the socio-ecological models of active living [23], Webber et al.'s model of mobility in older adults [107], the transportation and planning literature [108] and time geography [108, 109]. Overall, the effects of potential moderators shown in Fig. 2 are largely unexplored and constitute an important component of the research agenda for future studies.

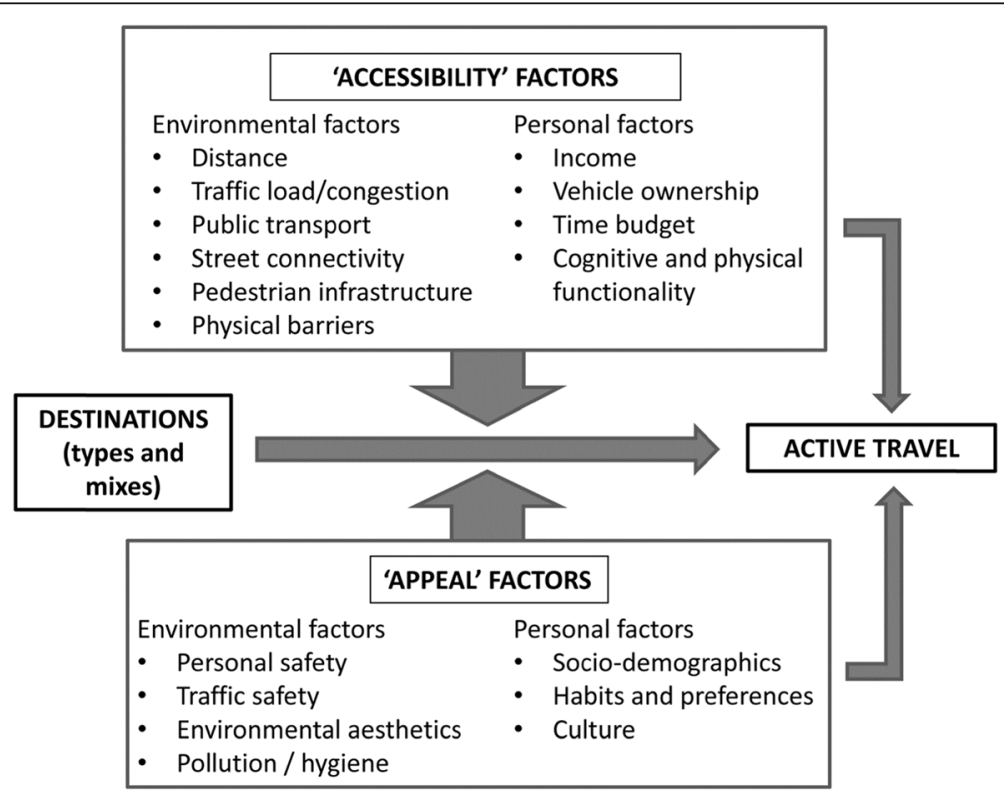

Fig. 2 A proposed conceptual framework of AAA+ destinations: Available, Accessible, Appealing for Active travel in an Ageing population 
The mediating role of perceptions of the neighbourhood environment in explaining the effects of the objective environment on AT is another critical issue (not depicted in Fig. 2) that needs to be tackled in future studies. Only one of the 42 reviewed studies investigated this matter [51]. If, as suggested by this synthesis of evidence, perceptions of destinations are more powerful predictors of AT than objective measures, it is important to understand the extent to which and under what circumstances perceptions may be manipulated through environmental changes.

Another contested issue regards the definition of neighbourhood or size of the area that influences older adults' AT behaviour. While self-report measures typically define a neighbourhood using a time metric (e.g., 15-20 min walk from home [110]), objective measures use distance (e.g., $400 \mathrm{~m}$ street-network buffer around the home). As the transportation literature suggests that most individuals are prepared to travel up to $20 \mathrm{~min}$ to discretionary destinations [69], it makes sense to define buffers around the home in terms of time. This is because the distance walked or cycled in a specific amount of time depends on one's physical capacity and the environmental characteristics of the street network.

In general, the majority of studies on environmental correlates of older adults' AT have been conducted in North America. There is a paucity of findings from other geographical regions. The same applies to cycling as a mode of transport, including the use of electric bicycles as a viable option for older adults with lower functional capacity [111]. Future studies need to address these knowledge gaps.

\section{Conclusions and implications for policy and practice}

Although research on the neighbourhood physical environmental correlates of older adults' AT has flourished in the last 5 years, more well-conducted studies across the globe are needed to establish the optimal profiles and thresholds of neighbourhood environmental attributes that support walking and cycling for transport in different groups of older adults. Acknowledging the limitations of the available evidence, this review suggests that in order to promote and support older adults' AT, especially walking for transport, it is important that neighbourhoods be walkable. Specifically, older residents should be provided easy, within-walkingdistance access to shops, public transport, recreational facilities and various commercial and institutional services through a network of well-maintained and safe footpaths with sufficient places to rest (i.e., benches). It is important that environmental enhancements be developed and implemented so that they are optimally matched to the context-specific needs (e.g., extant levels of density) and demographics (e.g., sex and age composition) of the community. Also, objective enhancements to the neighbourhood environment may need to be accompanied by efforts to raise awareness of these enhancements.

\section{Additional files}

Additional file 1: PRISMA checklist. (DOC 62 kb)

Additional file 2: Table S1. Neighbourhood physical environment and active travel in older adults - study characteristics and findings. (DOCX $87 \mathrm{~kb}$ )

Additional file 3: Supplementary analytical example - computation of $p$-values for associations of food outlets with all active travel outcomes in older adults. (DOCX $26 \mathrm{~kb}$ )

Additional file 4: Table S2. Article quality assessment. (DOCX 28 kb)

Additional file 5: Table S3. Summary table of meta-analytic results of significance of associations of neighbourhood built environmental correlates of active travel outcomes in older adults by type of adjustment for article characteristics. (DOCX $35 \mathrm{~kb}$ )

\section{Abbreviations}

AT: Active travel; CEPA: Council for the Environment and Physical Activity; GIS: Geographic Information Systems; GPS: Global Positioning System; NEWS: Neighborhood Environment Walkability Scale; PA: Physical activity; SD: Standard deviation; SES: Socio-economic status; UK: United Kingdom; USA: United States of America

\section{Acknowledgements}

This work has been undertaken as a collaborative effort under the umbrella of the Council on Environment and Physical Activity - Older Adults working group chaired by Ester Cerin.

\section{Funding}

Ester Cerin is supported by an Australian Research Council Future Fellowship FT3 140100085. The funding body had no role in the design of the review and meta-analysis, interpretation of data or writing of the manuscript.

\section{Availability of data and materials}

All information generated and analysed in this systematic review and meta-analysis are included in this published article and its supplementary information files (additional files).

\section{Authors' contribution}

EC conceptualised the systematic review, developed the review protocol and meta-analytical approach, conducted the meta-analyses, drafted the manuscript and supplementary material, extracted the data from the selected articles, search the grey literature and contributed to the selection of articles (screening for article eligibility). AN, JVC, DWB and AB conducted all searches, contributed to the screening for article eligibility and revised the manuscript for intellectual content. AB, AN and JVC verified the validity of the extracted data from the selected articles. AB drafted the introduction of the review. All authors approved the final manuscript.

\section{Competing interests}

The authors declare that they have no competing interests.

\section{Consent for publication}

Not applicable.

Ethical approval and consent to participate

Not applicable.

\section{Author details}

${ }^{1}$ Institute for Health and Ageing, Australian Catholic University, Level 6, 215 Spring Street, Melbourne, VIC 3000, Australia. ${ }^{2}$ School of Public Health, The University of Hong Kong, Hong Kong, China. ${ }^{3}$ Baker IDI Heart and Diabetes Institute, Melbourne, Australia. ${ }^{4}$ Department of Public Health, Ghent University, Ghent, Belgium. 
Received: 10 December 2016 Accepted: 31 January 2017 Published online: 06 February 2017

\section{References}

1. Jackson PA, Pialoux V, Corbett D, Drogos L, Erickson Kl, Eskes GA, et al. Promoting brain health through exercise and diet in older adults: a physiological perspective. J Physiol. 2016;594:4485-98. doi:10.1113/JP271270.

2. World Health Organisation. Global recommendations on physical activity for health. Geneva: WHO Press; 2010. http://www.who.int/dietphysicalactivity/ global-PA-recs-2010.pdf Accessed 13 Oct 2016

3. Kelly P, Kahlmeier S, Götschi T, Orsini N, Richards J, Roberts N, et al. Systematic review and meta-analysis of reduction in all-cause mortality from walking and cycling and shape of dose response relationship. Int J Behav Nutr Phys Act. 2014;11:132. doi:10.1186/s12966-014-0132-x.

4. Barnett A, Cerin E, Zhang CJ, Sit CH, Johnston JM, Cheung MM, et al. Associations between the neighbourhood environment characteristics and physical activity in older adults with specific types of chronic conditions: the ALECS cross-sectional study. Int J Behav Nutr Phys Act. 2016;13:53. doi:10.1186/s12966-016-0377-7.

5. Mendes de Leon CF, Cagney KA, Bienias JL, Barnes LL, Skarupski KA, Scherr PA, et al. Neighborhood social cohesion and disorder in relation to walking in community-dwelling older adults: a multilevel analysis. J Aging Health. 2009;21:155-71. doi:10.1177/0898264308328650.

6. Van Holle V, Van Cauwenberg J, Van Dyck D, Deforche B, Van de Weghe N, De Bourdeaudhuij I. Relationship between neighborhood walkability and older adults' physical activity: results from the Belgian Environmental Physical Activity Study in Seniors (BEPAS Seniors). Int J Behav Nutr Phys Act. 2014;11:110. doi:10.1186/s12966-014-0110-3.

7. Sahlqvist S, Goodman A, Cooper AR, Ogilvie D, on behalf of the iConnect consortium. Change in active travel and changes in recreational and total physical activity in adults: longitudinal findings from the iConnect study. Int J Behav Nutr Phys Act. 2013;10:28.

8. Foley L, Panter J, Heinen E, Prins R, Ogilvie D. Changes in active commuting and changes in physical activity in adults: a cohort study. Int J Behav Nutr Phys Act. 2015;12:161. doi:10.1186/s12966-015-0323-0.

9. Mueller N, Rojas-Rueda D, Cole-Hunter T, de Nazelle Al, Dons E, Gerike R, et al. Health impact assessment of active transportation: a systematic review. Prev Med. 2015;76:103-14. doi:10.1016/j.ypmed.2015.04.010

10. Sallis JF, Spoon C, Cavill N, Engelberg JK, Gebel K, Parker M, et al. Co-benefits of designing communities for active living: an exploration of literature. Int J Behav Nutr Phys Act. 2015;12:30. doi:10.1186/s12966-015-0188-2.

11. Woodcock J, Edwards P, Tonne C, Armstrong BG, Ashiru O, Banister D, et al. Public health benefits of strategies to reduce greenhouse-gas emissions: urban land transport. Lancet. 2009;374:1930-43.

12. Grabow ML, Spak SN, Holloway T, Stone B Jr, Mednick AC, Patz JA. Air quality and exercise-related health benefits from reduced car travel in the midwestern United States. Environmental Health Perspectives, Epub. 2 November 2011.

13. Jarrett J, Woodcock J, Griffiths UK, Chalabi Z, Edwards P, Roberts I, Haines A. Effect of increasing active travel in urban England and Wales on costs to the National Health Service. Lancet. 2012;379:2198-205. doi:10.1016/S0140-6736(09)61714-1.

14. Tainio M, de Nazelle AJ, Götschi T, Kahlmeier S, Rojas-Rueda D, Nieuwenhuijsen MJ, et al. Can air pollution negate the health benefits of cycling and walking? Prev Med. 2016;87:233-6. http://dx.doi.org/10.1016/j. ypmed.2016.02.002.

15. Franco MR, Tong A, Howard K, Sherrington C, Ferreira PH, Pinto RZ, et al. Older people's perspectives on participation in physical activity: a systematic review and thematic synthesis of qualitative literature. Br J Sports Med. 2015:49:1268-76. doi:10.1136/bjsports-2014-094015.

16. Berkemeyer K, Wijndaele K, White T, Cooper AJ, Luben R, Westgate K, et al. The descriptive epidemiology of accelerometer-measured physical activity in older adults. Int J Behav Nutr Phys Act. 2016;13:2. doi:10.1186/s12966-015-0316-z.

17. Keadle SK, McKinnon R, Graubard BI, Troiano RP. Prevalence and trends in physical activity among older adults in the United States: a comparison across three national surveys. Prev Med. 2016;89:37-43. doi:10.1016/j.ypmed. 2016.05.009.

18. Laverty AA, Palladino R, Lee JT, Millett C. Associations between active travel and weight, blood pressure and diabetes in six middle income countries: a cross-sectional study in older adults. Int J Behav Nutr Phys Act. 2015;12:65. doi:10.1186/s12966-015-0223-3.
19. Van Cauwenberg J, De Bourdeaudhuij I, De Meester F, Van Dyck D, Salmon J, Clarys P, et al. Relationship between the physical environment and physical activity in older adults: a systematic review. Health Place. 2011;17:458-69. doi:10.1016/j.healthplace.2010.11.010.

20. Davison KK, Lawson CT. Do attributes in the physical environment influence children's physical activity? A review of the literature. Int J Behav Nutr Phys Act. 2006;3:19. doi:10.1186/1479-5868-3-19.

21. Barnes LL, Wilson RS, Bienias JL, Mendes de Leon CF, Kim H-JM, Buchman AS, et al. Correlates of life space in a volunteer cohort of older adults. Exp Aging Res. 2007;33:77-93. doi:10.1080/03610730601006420

22. Ding D, Sallis JF, Norman GJ, Frank LD, Saelens BE, Kerr J, et al. Neighborhood environment and physical activity among older adults: do the relationships differ by driving status? J Aging Phys Activ. 2014;22:421-31. doi:10.1123/japa.2012-0332.

23. Sallis J, Cervero R, Ascher W, Henderson K, Kraft M, Kerr J. An ecological approach to creating active living communities. Annu Rev of Public Health. 2006;27:297-322. doi:10.1146/annurev.publhealth.27.021405.102100.

24. Kerr J, Emond JA, Badland H, Reis R, Sarmiento O, Carlson J, et al. Perceived neighborhood environmental attributes associated with walking and cycling for transport among adult residents of 17 cities in 12 countries: the IPEN study. Environ Health Perspect. 2016;124:290-8. doi:10.1289/ehp.1409466.

25. Van Cauwenberg J, Clarys P, De Bourdeaudhuij I, Van Holle V, Verté D, De Witte $\mathrm{N}$, et al. Physical environmental factors related to walking and cycling in older adults: the Belgian aging studies. BMC Public Health. 2012;12:142. doi:10.1186/1471-2458-12-142.

26. Gebel K, Ding D, Foster C, Bauman AE, Sallis JF. Improving current practice in reviews of the built environment and physical activity. Sports Med. 2015;45:297-302. doi:10.1007/s40279-014-0273-8.

27. Cerin E, Barnett A, Barnett D, Nathan A, Van Cauwenberg J. Systematic review and meta-analysis of the built environment and active travel in older adults. PROSPERO 2016:CRD42016046818.

28. Moher D, Liberati A, Tetzlaff J, Altman DG, PRISMA Group. Preferred reporting items for systematic reviews and meta-analyses: the PRISMA statement. PLoS Med. 2009;6:e1000097. doi:10.1371/journal.pmed.1000097.

29. Cerin E, Conway TL, Cain KL, Kerr J, De Bourdeaudhuij I, Owen N, et al. Sharing good NEWS across the world: developing comparable scores across 12 countries for the Neighborhood Environment Walkability Scale (NEWS). BMC Public Health. 2013;13:309. doi:10.1186/1471-2458-13-309.

30. Cerin E, Sit CH, Cheung MC, Ho SY, Lee LC, Chan WM. Reliable and valid NEWS for Chinese seniors: measuring perceived neighborhood attributes related to walking. Int J Behav Nutr Phys Act. 2010;7:84. doi:10.1186/1479-5868-7-84.

31. Saelens BE, Sallis JF, Black JB, Chen D. Neighborhood-based differences in physical activity: an environment scale evaluation. Am J Public Health. 2003;93(9):1552-8.

32. Cain KL, Millstein RA, Sallis JF, Conway TL, Gavand KA, Frank LD, et al. Contribution of streetscape audits to explanation of physical activity in four age groups based on the Microscale Audit of Pedestrian Streetscapes (MAPS). Soc Sci Med. 2014;116:82-92. doi:10.1016/j. socscimed.2014.06.042.

33. Etman A, Kamphuis CB, Prins RG, Burdorf A, Pierik FH, van Lenthe FJ. Characteristics of residential areas and transportational walking among frail and non-frail Dutch elderly: does the size of the area matter? Int J Health Geogr. 2014;13:7. doi:10.1186/1476-072X-13-7.

34. King AC, Sallis JF, Frank LD, Saelens BE, Cain K, Conway TL, et al. Aging in neighborhoods differing in walkability and income: associations with physical activity and obesity in older adults. Soc Sci Med. 2011;73:1525-33. doi:10.1016/j.socscimed.2011.08.032.

35. Inoue S, Ohya Y, Odagiri Y, Takamiya T, Kamada M, Okada S, et al. Perceived neighborhood environment and walking for specific purposes among elderly Japanese. J Epidemiol. 2011;21:481-90. doi:10.2188/jea.JE20110044.

36. Ewing R, Cervero R. Travel and the built environment. J Am Plan Assoc. 2010;73(3):265-94. doi:10.1080/01944361003766766.

37. Ogilvie D, Foster CE, Rothnie H, Cavill N, Hamilton H, Fitzsimons CF, et al. Interventions to promote walking: systematic review. BMJ. 2007;334:1204. doi:10.1136/bmj.39198.722720.BE.

38. Kerr J, Sallis JF, Owen N, De Bourdeaudhuij I, Cerin E, Sugiyama T, et al. Advancing science and policy through a coordinated international study of physical activity and built environments: IPEN adult methods. J Phys Act Health. 2013;10(4):581-601.

39. Cerin E. Statistical approaches to testing the relationships of the built environment with resident-level physical activity behavior and health 
outcomes in cross-sectional studies with cluster sampling. J Plann Lit. 2011;26:151-67. doi:10.1177/0885412210386229.

40. Lamb KE, White SR. Categorisation of built environment characteristics: the trouble with tertiles. Int J Behav Nutr Phys Act. 2015;12:19. doi:10.1186/ s12966-015-0181-9.

41. Nathan A, Wood L, Giles-Corti B. Exploring socioecological correlates of active living in retirement village residents. J Aging Phys Act. 2014;22(1):1-15. doi:10.1123/japa.2012-0189.

42. Cohen J. Statistical power analysis for the behavioural sciences. 2nd ed. Hillsdale: Erlbaum; 1989.

43. Davey J, Turner RM, Clarke MJ, Higgins JP. Characteristics of meta-analyses and their component studies in the Cochrane Database of Systematic Reviews: a cross-sectional, descriptive analysis. BMC Med Res Methodol. 2011;11:160. doi:10.1186/1471-2288-11-160.

44. Higgins JPT, Thompson SG, Spiegelhalter DJ. A re-evaluation of random effects meta-analysis. J R Stat Soc A Stat Soc. 2009;172:137-59. doi:10.1111/j.1467-985X.2008.00552.x.

45. Rosenthal R. New directions for methodology of social and behavioural science: quantitative assessment of research domains. San Francisco: JosseyBass; 1980.

46. Bland M. An introduction to medical statistics. 3rd ed. Oxford: University Press; 2000.

47. Kolbe-Alexander TL, Pacheco K, Tomaz SA, Karpul D, Lambert EV. The relationship between the built environment and habitual levels of physical activity in South African older adults: a pilot study. BMC Public Health. 2015;15:518. doi:10.1186/s12889-015-1853-8.

48. Maisel JL. Impact of older adults' neighborhood perceptions on walking behaviour. J Aging Phys Act. 2016;24:247-55. doi:10.1123/japa.2014-0278.

49. Patterson PK, Chapman NJ. Urban form and older residents' service use, walking, driving, quality of life, and neighborhood satisfaction. Am J Health Promot. 2004;19:45-52. doi:10.4278/0890-1171-19.1.45.

50. Sugiyama T, Ward Thompson C. Associations between characteristics of neighbourhood open space and older people's walking. Urban For Urban Greening. 2008;7:41-51.

51. King D. Neighborhood and individual factors in activity in older adults: results from the Neighborhood and Senior Health Study. J Aging Phys Act. 2008;16:144-70.

52. King AC, Toobert D, Ahn D, Resnicow K, Coday M, Riebe D, et al. Perceived environments as physical activity correlates and moderators of intervention in five studies. Am J Health Promot. 2006;21:24-35.

53. Davis MG, Fox KR, Hillsdon M, Coulson JC, Sharp DJ, Stathi A, et al. Getting out and about in older adults: the nature of daily trips and their association with objectively assessed physical activity. Int J Behav Nutr Phys Act. 2011;8:116. doi:10.1186/1479-5868-8-116.

54. Chudyk AM, Winters M, Moniruzzaman M, Ashe MC, Gould JS, McKay H. Destinations matter: the association between where older adults live and their travel behavior. J Transp Health. 2015;2(1):50-7. doi:10.1016/j.jth.2014.09.008.

55. Mitchell $A B$. The relationship of self-reported health status and perceived neighborhood built environment with the amount of self-reported walking in community-dwelling older adults. Ann Arbor, Ml: ProQuest LLC 2012. Thesis. http://repository.upenn.edu/dissertations/AAI3542931.

56. Cerin E, Macfarlane D, Sit CHP, Ho SY, Johnston JM, Chou KL, et al. Effects of built environment on walking among Hong Kong older adults. Hong Kong Med J. 2013;19:S39-41.

57. Cerin E, Lee KY, Barnett A, Sit CH, Cheung MC, Chan WM, et al. Walking for transportation in Hong Kong Chinese urban elders: a cross-sectional study on what destinations matter and when. Int J Behav Nutr Phys Act. 2013;10:78. doi:10.1186/1479-5868-10-78.

58. Cerin E, Sit CH, Barnett A, Johnston JM, Cheung MC, Chan WM. Ageing in an ultra-dense metropolis: perceived neighbourhood characteristics and utilitarian walking in Hong Kong elders. Public Health Nutr. 2014;17:225-32. doi:10.1017/S1368980012003862.

59. Corseuil MW, Schneider IJ, Silva DA, Costa FF, Silva KS, Borges LJ, et al. Perception of environmental obstacles to commuting physical activity in Brazilian elderly. Prev Med. 2011;53:289-92. doi:10.1016/j.ypmed.2011.07.016.

60. Nathan A, Wood L, Giles-Corti B. Perceptions of the built environment and associations with walking among retirement village residents. Environ Behav. 2014;46:46-69. doi:10.1123/japa.2012-0189.

61. Van Cauwenberg J, Van Holle V, De Bourdeaudhuii I, Van Dyck D, Deforche B. Neighborhood walkability and health outcomes among older adults: the mediating role of physical activity. Health Place. 2016;37:16-25. doi:10.1016/j.healthplace.2015.11.003.

62. Barnes R, Winters M, Ste-Marie N, McKay H, Ashe MC. Age and retirement status differences in associations between the built environment and active travel behaviour. J Transp Health. 2016. doi:10.1016/j.jth.2016.03.003.

63. Shigematsu R, Sallis JF, Conway TL, Saelens BE, Frank LD, Cain KL, et al. Age differences in the relation of perceived neighborhood environment to walking. Med Sci Sports Exerc. 2009;41:314-21. doi:10.1249/MSS. Ob013e318185496c

64. Tsai LT, Rantakokko M, Portegijs E, Viljanen A, Saajanaho M, Eronen J, et al. Environmental mobility barriers and walking for errands among older people who live alone vs. with others. BMC Public Health. 2013;13:1054. doi:10.1186/1471-2458-13-1054.

65. Carlson JA, Sallis JF, Conway TL, Saelens BE, Frank LD, Kerr J, et al. Interactions between psychosocial and built environment factors in explaining older adults' physical activity. Prev Med. 2012;54:68-73. doi:10.1016/j.ypmed.2011.10.004.

66. Bracy NL, Millstein RA, Carlson JA, Conway TL, Sallis JF, Saelens BE, et al. Is the relationship between the built environment and physical activity moderated by perceptions of crime and safety? Int J Behav Nutr Phys Act. 2014;11:24. doi:10.1186/1479-5868-11-24.

67. Frank L, Kerr J, Rosenberg D, King A. Healthy aging and where you live: community design relationships with physical activity and body weight in older Americans. J Phys Act Health. 2010;7:S82-90.

68. World Health Organization. Global age-friendly cities: a guide. Geneva: WHO; 2007. http://www.who.int/ageing/age_friendly_cities_guide/en/

69. Department of Infrastructure and transport. Walking, riding and access to public transport: supporting active travel in Australian communities. Canberra: Commonwealth of Australia; 2013. https://infrastructure.gov.au/ infrastructure/pab/active_transport/files/infra1874_mcu_active_travel_ report_final.pdf.

70. Moniruzzaman M, Paez A, Scott D, Morency C. Trip generation of seniors and the geography of walking in Montreal. Environ Plann A. 2015;47:957-76. doi:10.1068/a130070p.

71. Christiansen LB, Cerin E, Badland H, Kerr J, Davey R, Troelsen J, et al. International comparisons of the associations between objective measures of the built environment and transport-related walking and cycling: IPEN adult study. J Transp Health. 2016;3:467-78. doi:10.1016/j.jth.2016.02.010.

72. Leslie E, Cerin E, du Toit L, Owen N, Bauman A. Objectively assessing 'walkability' of local communities: using GIS to identify the relevant environmental attributes. In: Lai PC, Mak AS, editors. GIS for Health and the Environment: Development in the Asia-Pacific Region. Berlin: Springer; 2007. p. 91-104.

73. Alfonzo MA. To walk or not to walk? The hierarchy of walking needs. Environ Behav. 2005:37:808-36. doi:10.1177/0013916504274016.

74. O'Hern S, Oxley J. Understanding travel patterns to support safe active transport for older adults. J Transp Health. 2015;2:79-85. http://dx.doi.org/10.1016/j.th.2014.09.016.

75. Barnett A, Cerin E, Cheung MC, Chan WM. An in-depth pilot study on patterns, destinations, and purposes of walking in Hong Kong older adults. J Aging Phys Act. 2015;23(1):144-52. doi:10.1123/japa.2013-0026.

76. Cerin E, Zhang CJ, Barnett A, Sit CH, Cheung MM, Johnston JM, Lai PC, et al. Associations of objectively-assessed neighborhood characteristics with older adults' total physical activity and sedentary time in an ultra-dense urban environment: findings from the ALECS study. Health Place. 2016:42:1-10. doi:10.1016/j.healthplace.2016.08.009.

77. Moran M, Van Cauwenberg J, Hercky-Linnewiel R, Cerin E, Deforche B, Plaut P. Understanding the relationships between the physical environment and physical activity in older adults: a systematic review of qualitative studies. Int J Behav Nutr Phys Act. 2014;11:79. doi:10.1186/1479-5868-11-79.

78. Van Cauwenberg J, De Bourdeaudhuij I, Clarys P, Nasar J, Salmon J, Goubert L, et al. Street characteristics preferred for transportation walking among older adults: a choice-based conjoint analysis with manipulated photographs. Int J Behav Nutr Phys Act. 2016;13:6. doi:10.1186/s12966-016-0331-8.

79. Dunlop DD, Song J, Semanik PA, Chang RW, Sharma L, Bathon JM, et al. Objective physical activity measurement in the osteoarthritis initiative: are guidelines being met? Arthritis Rheum. 201 1;63:3372-82. doi:10.1002/art.30562.

80. Willis JR, Jefferys $\mathrm{JL}$, Vitale S, Ramulu PY. Visual impairment, uncorrected refracted error, and accelerometer-defined physical activity in the United States. Arch Opthamol. 2012;130:1329-35. doi:10.1001/archopthalmol.2011. 1773. 
81. Van Holle V, Deforche B, Van Cauwenberg J, Goubert L, Maes L, Van de Weghe N, et al. Relationship between the physical environment and different domains of physical activity in European adults: a systematic review. BMC Public Health. 2012;12:807. doi:10.1186/1471-2458-12-807.

82. Witten K, Blakely T, Bagheri N, Badland H, Ivory V, Pearce J, et al. Neighborhood built environment and transport and leisure physical activity: findings using objective exposure and outcome measures in New Zealand. Environ Health Perspect. 2012;120:971-7. doi:10.1289/ehp.1104584

83. Cerin E, Chan KW, Macfarlane DJ, Lee KY, Lai PC. Objective assessment of walking environments in ultra-dense cities: development and reliability of the Environment in Asia Scan Tool-Hong Kong version (EAST-HK). Health Place. 2011;17:937-45. doi:10.1016/j.healthplace.2011.04.005.

84. Foster S, Giles-Corti B. The built environment, neighborhood crime and constrained physical activity: an exploration of inconsistent findings. Prev Med. 2008:47:241-51. doi:10.1016/j.ypmed.2008.03.017.

85. Cerin E, Cain KL, Conway TL, Van Dyck D, Hinckson E, Schipperijn J, et al. Neighborhood environments and objectively measured physical activity in 11 countries. Med Sci Sports Exerc. 2014;46:2253-64. doi:10.1249/MSS. 0000000000000367.

86. Cerin E, Barnett A, Sit CH, Cheung MC, Lee LC, Ho SY, et al. Measuring walking within and outside the neighborhood in Chinese elders: reliability and validity. BMC Public Health. 2011;11:851. doi:10.1186/1471-2458-11-851

87. Giles-Corti B, Timperio A, Bull F, Pikora T. Understanding physical activity environmental correlates: increased specificity for ecological models. Exerc Sport Sci Rev. 2005;33(4):175-81.

88. Nathan A, Wood L, Giles-Corti B. Examining correlates of self-reported and objectively measured physical activity among retirement village residents. Australas J Ageing. 2014;33:250-6. doi:10.1111/ajag.12055.

89. Moniruzzaman M, Paez A, Khandker M, Habib N, Morency C. Mode use and trip length of seniors in Montreal. J Transp Geogr. 2013;30:89-99. http://dx.doi.org/10.1016/j.jtrangeo.2013.03.007.

90. Corseuil Giehl MW, Hallal PC, Corseuil CW, Schneider IJ, d'Orsi E. Built environment and walking behavior among Brazilian older adults: a population-based study. J Phys Act Health. 2016;13:617-24. doi:10.1123/jpah.2015-0355.

91. Procter-Gray E, Leveille SG, Hannan MT, Cheng J, Kane K, Li W. Variations in community prevalence and determinants of recreational and utilitarian walking in older age. J Aging Res. 2015;2015:382703. doi:10.1155/2015/382703.

92. Boruff BJ, Nathan A, Nijënstein S. Using GPS technology to (re)-examine operational definitions of 'neighbourhood' in place-based health research. Int J Health Geogr. 2012;11:22. doi:10.1186/1476-072X-11-22.

93. Nyunt MS, Shuvo FK, Eng JY, Yap KB, Scherer S, Hee LM, et al. Objective and subjective measures of neighborhood environment (NE): relationships with transportation physical activity among older persons. Int J Behav Nutr Phys Act. 2015;12:108. doi:10.1186/s12966-015-0276-3.

94. Corseuil Giehl MW, Hallal PC, Brownson RC, d'Orsi E. Exploring associations between perceived measures of the environment and walking among Brazilian older adults. J Aging Health. 2016.

95. Pelclová J, Frömel K, Bláha L, Zajac-Gawlak I, Tlucakova L. Neighborhood environment and walking for transport and recreation in Central European older adults. Acta Univ Palacki Olomouc Gymn. 2012;42:49-56. doi:10.5507/ag.2012.023.

96. Menz HB, Lord SR, Fitzpatrick RC. Age-related differences in walk stability. Age Ageing. 2003;32:137-42.

97. Garrard J. Senior Victorians and walking: obstacles and opportunities: summary report. Melbourne: Victoria Walks Inc.; 2013. http://www. victoriawalks.org.au/Assets/Files/FINALSeniorsSummary.pdf.

98. Cao X, Mokhtarian P, Handy S. Examining the impacts of residential selfselection on travel behaviour: a focus on empirical findings. Transport Rev. 2009;29:359-95. http://dx.doi.org/10.1080/01441640802539195.

99. McCormack GR, Shiell A. In search of causality: a systematic review of the relationship between the built environment and physical activity among adults. Int J Behav Nutr Phys Act. 2011;8:125. doi:10.1186/1479-5868-8-125.

100. Martin A, Ogilvie D, Suhrcke M. Evaluating causal relationships between urban built environment characteristics and obesity: a methodological review of observational studies. Int J Behav Nutr Phys Act. 2014;11:142 doi:10.1186/s12966-014-0142-8.

101. Boone-Heinonen J, Guilkey D, Evenson K, Gordon-Larsen P. Residential selfselection bias in the estimation of built environment effects on physical activity between adolescence and young adulthood. Int J Behav Nutr Phys Act. 2010;7:70. doi:10.1186/1479-5868-7-70.
102. Dillman DA. Mail and internet surveys: the tailored design method Chichester. New York: Wiley; 2000.

103. Burton NW, Haynes M, Wilson LA, Giles-Corti B, Oldenburg BF, Brown WJ, et al. HABITAT: a longitudinal multilevel study of physical activity change in mid-aged adults. BMC Public Health. 2009;9:76. doi:10.1186/1471-2458-9-76.

104. Kelly BJ, Fraze TK, Hornik RC. Response rates to a mailed survey of a representative sample of cancer patients randomly drawn from the Pennsylvania Cancer Registry: a randomized trial of incentive and length effects. BMC Med Res Methodol. 2010;10:65. doi:10.1186/1471-2288-10-65.

105. Chaix B, Méline J, Duncan S, Merrien C, Karusisi N, Perchoux C, et al. GPS tracking in neighborhood and health studies: a step forward for environmental exposure assessment, a step backward for causal inference? Health Place. 2013;21:46-51. doi:10.1016/j.healthplace.2013.01.003.

106. Takemoto M, Carlson JA, Moran K, Godbole S, Crist K, Kerr J. Relationship between objectively measured transportation behaviors and health characteristics in older adults. Int J Environ Res Public Health. 2015;12:13923-37. doi:10.3390/ijerph121113923.

107. Webber SC, Porter MN, Menec VH. Mobility in older adults: a comprehensive framework. Gerontologist. 2010;50:443-50. doi:10.1093/geront/gnq013.

108. Handy SL, Niemeier DA. Measuring accessibility: an exploration of issues and alternatives. Environ Plann A. 1997;29:1175-94. doi:10.1068/a291175.

109. Rainham D, McDowell I, Krewski D, Sawada M. Conceptualizing the healthscape: contributions of time geography, location technologies and spatial ecology to place and health research. Soc Sci Med. 2010;70:668-76. doi:10.1016/j.socscimed.2009.10.035.

110. Van Holle V, Van Cauwenberg J, Gheysen F, Van Dyck D, Deforche B, Van de Weghe $\mathrm{N}$, et al. The association between Belgian older adults' physical functioning and physical activity: what is the moderating role of the physical environment? PLoS One. 2016;11:e0148398. doi:10.1371/journal. pone.0148398.

111. Johnson $M$, Rose $G$. Extending life on a bike: electric bike use by older Australians. J Transp Health. 2015;2:276-83. doi:10.1016/j.jth.2015.03.001.

\section{Submit your next manuscript to BioMed Central and we will help you at every step:}

- We accept pre-submission inquiries

- Our selector tool helps you to find the most relevant journal

- We provide round the clock customer support

- Convenient online submission

- Thorough peer review

- Inclusion in PubMed and all major indexing services

- Maximum visibility for your research

Submit your manuscript at www.biomedcentral.com/submit

) Biomed Central 